DOE-ER-03992-532

Aucust 1983

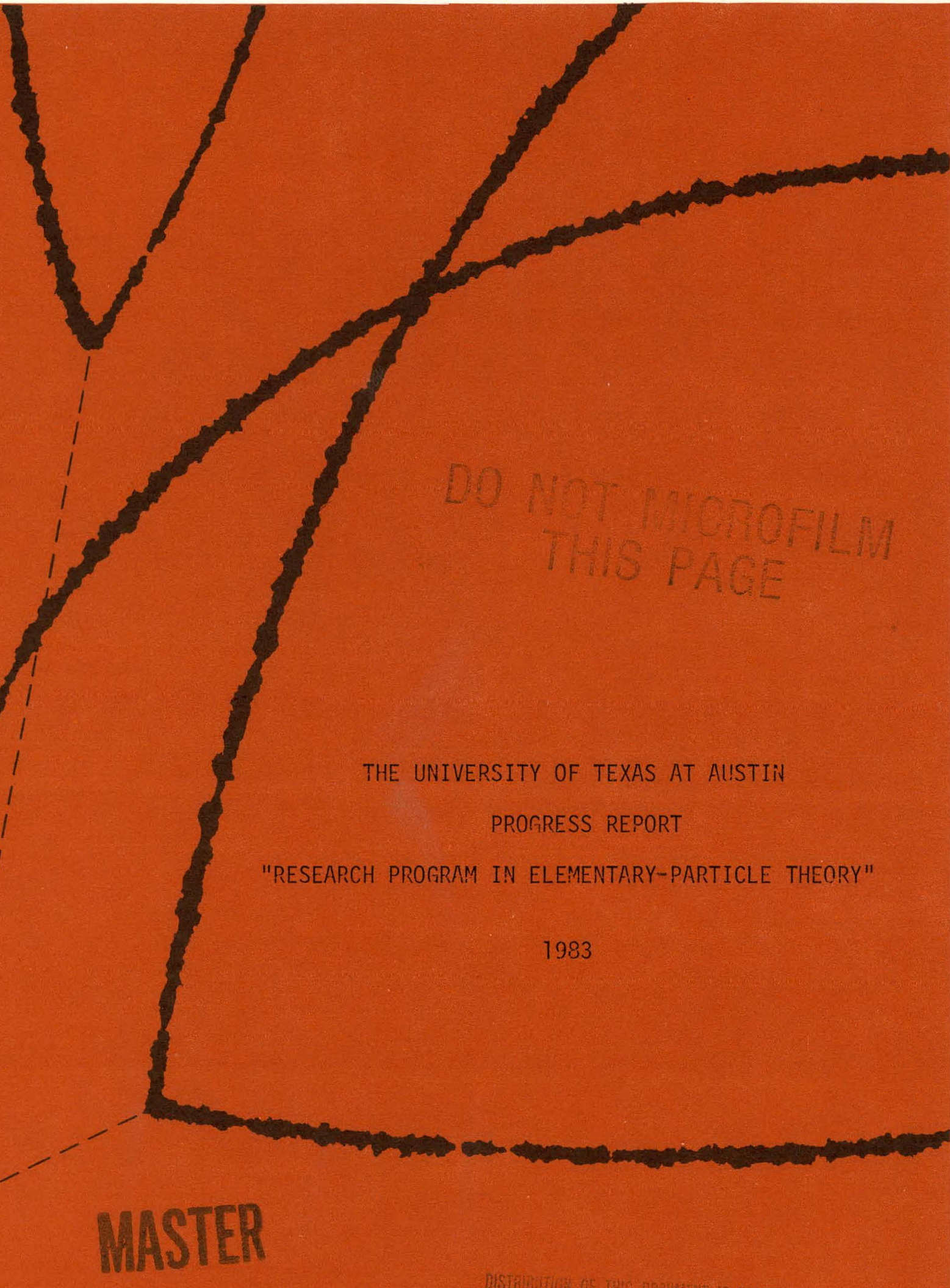

The Center for Particle Theory

THE UNIVERSITY OF TEXAS AT AUSTIN 


\section{DISCLAIMER}

This report was prepared as an account of work sponsored by an agency of the United States Government. Neither the United States Government nor any agency Thereof, nor any of their employees, makes any warranty, express or implied, or assumes any legal liability or responsibility for the accuracy, completeness, or usefulness of any information, apparatus, product, or process disclosed, or represents that its use would not infringe privately owned rights. Reference herein to any specific commercial product, process, or service by trade name, trademark, manufacturer, or otherwise does not necessarily constitute or imply its endorsement, recommendation, or favoring by the United States Government or any agency thereof. The views and opinions of authors expressed herein do not necessarily state or reflect those of the United States Government or any agency thereof. 


\section{DISCLAIMER}

Portions of this document may be illegible in electronic image products. Images are produced from the best available original document. 


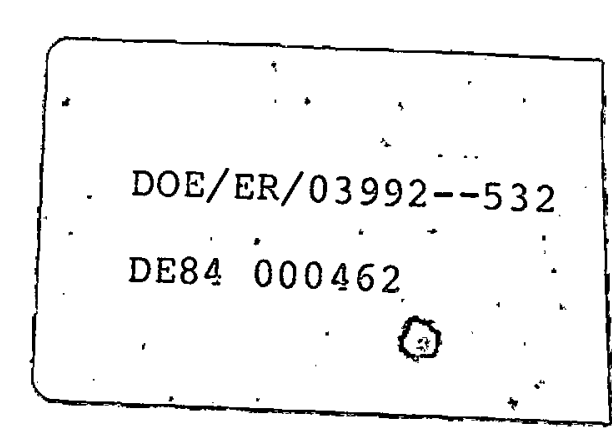

\title{
THE UNIVERSITY OF TEXAS AT AUSTIN
}

Progress Report

on

"RESEARCH PROGRAM IN ELEMENTARY-PARTICLE THEORY",

1983. अ

\author{
Submitted to \\ Division of Research \\ Department of Energy \\ Washington, D. C. 20545
}

by

E. C. G. Sudarshan

and

Y. Ne'eman

Center for Particle Theory

Physics Department

Austin, Texas 78712

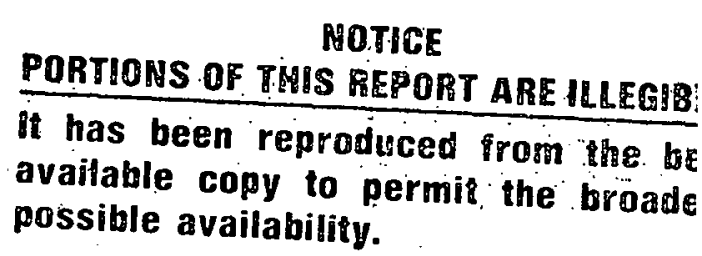

DISCLAIMER

This report was prepared as an account of work sponsored by an agency of the United States Government. Neither the United States Government nor any agency thereof, nor any of their employees, makes any warranty, express or implied, or assumes any legal liahility or resmnsibility for the accuracy, completeness, or usefulness of any information, apparatus, product, or process disclosed, or represents that its use would not infringe privately owned rights. Reference herein to any specific commercial product, process, or service by trade name, trademark, manufacturer, or otherwise does not necessarily constitute or imply its endorsement, recommendation, or favoring by the United States Government or any agency thereof. The views and opinions of authors expressed herein do not necessarily state or reflect those of the United States Government or any agency thereof.

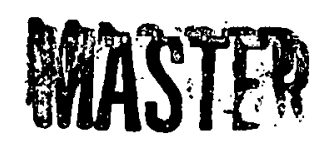


TABLE OF CONTENTS

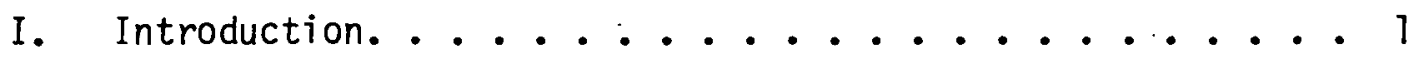

II. Research. Report

A. Physics of UTtra High Energies and Cosmology. . . . 2

B. Phenomenology of Particle Physics ........7

C. Quantum Field Theory, Supersymmetry and Models of Particles. ................... 14

D. Geometric Formulations and ATgebraic Models .... 20

APPENDIX A - Titles of DOE Reports Since DOE-ER-03992-496. . . A23

APPENDIX B - Research Histories of the Scientific Staff of the Center for Particle Theory. ....... B27 
I. INTRODUCTION

This progress report of the Center for Particle Theory of the University of Texas at Austin reviews the work done over the past year and is part of the renewal proposal for the period from January 1, 1984 to December 31, 1984.

The senior academic staff of the Center for Particle Theory has essentially remained the same. Professor Ne'eman had to rearrange his schedule to accommodate his increased responsibifities in Israel. Dr. Tata is leaving the group for the University of Oregon. Dr. S. Nandi continues as a member of the staff. Professor" $P$. Kielanowski has been a visiting member of the group working in close association with Professor A. Bohm. It is expected that his collaboration will continue. Dr. Allen Stern has returned from a year's leave of absence at the University of Goteborg, Sweden.

Professor Sudarshan visited the Peoples Republic of China by invitation of the Academia Sinica and lectured at various institutions: in Beijing, Souzhou and Shanghai but spent most of his time at the Institute of Theoretical Physics of the Academia Sinica in Beijing. 


\section{RESEARCH REPORT}

A. Physics of Ultra High Energies and Cosmology

The standard hot big-bang model of the universe seems to provide a reliable framework for understanding the evolution of the large scale structure of our universe. One of the features of our present universe which is naturally explained in this model is the large abundance of ${ }^{4} \mathrm{He}$. The success of primordial nucleosynthesis in predicting the large primordial abundance of ${ }^{4} \mathrm{He}$, and the relatively large abundance of $D$, is usually considered to be the strongest evidence that the universe can be described by a Friedmann-Robertson-Walker cosmology at very early times. Because of this concordance, it is attractive to assume that the Friedmann-Robertson-Walker cosmology was applicable at the time of nucleosynthesis, and then demand that the resulting primordial abundances of the light elements be within bounds extrapolated from present observations. Thus it is important to have precise predictions of the primordial light element abundances, particularly that of ${ }^{4} \mathrm{He}$.

In a paper titled "Primordial Nucleosynthesis Including Radiative, Coulomb, and Finite Temperature Corrections to Weak Rates" (DOE-ER-03992-494) Drs. Dicus, Gleeson and Sudarshan, together with E. Kolb of Los Alamos Scientific Lab, V. Teplitz of the Arms Control Agency and M. Turner of the University of Chicago expand on an earlier suggestion of S. Glashow and Sudarshan that the masses of particles are shifted by temperature effects to calculate the temperature and density effects on the abundance of 4 He. Modifications to the calculation of the ${ }^{4} \mathrm{He}$ abundance include 1) explicit numerical integration of the rates for $n-p$ transitions rather. than fits to the numerical rates; 2) correct treatment of Coulomb corrections; 3 ) inclusion of radiative corrections--both the usual radiative corrections, and the finite temperature and finite density radiative corrections that depend on the presence of a plasma; 4) inclusion of the effect of the plasma on the mass of the electron; and 5 ) heating of electron neutrinos in $\mathrm{e}^{+} \mathrm{e}^{-}$annihilation. The result is that the above five effects result in a systematic decrease in the ${ }^{4} \mathrm{He}$ abundance of about 0.003 , or about a one percent relative decrease. There are similar $1-2 \%$ changes in the abundances of the other light elements which are produced $\left(0,{ }^{3} \mathrm{He},{ }^{7} \mathrm{Li}\right.$, etc.). This work was also reported to the Telemark Neutrino Conference in September, 1982. 
In a related paper "On Perturbation Theory at Finite Temperature" (DOE-ER-03992-507) Dicus, together with a graduate student David Down, and E. Kolb of Los Alamos calculated to order $\alpha^{2}$ the shift of the mass of a charged fermion due to its interaction with a plasma at non-zero temperature (but zero chemical potential) and its effect on the physical quantities of the number and energy density. At finite temperature to order $\alpha^{2}$, the largest contribution of the fermion self energy to the number density or energy density comes from terms in the energy squared which grows as $T$. Since these go as $\alpha^{2} T^{4} / p^{2}$ for external 3-momentum $p$ not equa to zero (neglecting logarithm in $\mathrm{p} / \mathrm{m})$, rather than as $\alpha^{2} T^{4} / \mathrm{m}_{0}^{2}$, the energy density and number densities are essentiall unchanged. Further, they have verified for two loops that the self energy is a power series in temperature, $\Sigma_{B} \sim \alpha^{n} T^{n+1}\left(p^{-1} \ln \frac{E+p}{E-p}\right)^{n}$ and thus never significantly aiters physical quantities such as the number density or energy density.

A macroscopic model for the dynamics of stellar collapse is impossible without a complete understanding of the nuclear and particle microphysics that describes the interactions of the particles... For instance, unified models of weak and electromagnetic interactions predict that neutrinos should scatter coherently with nuclei... As a consequence of coherent scattering, neutrinos are trapped in the latter stages of the collapse of massive stars and this trapping of neutrinos is a crucial ingredient in the present theory of supernovae. Aithough the Weinberg-Salam model may not be the ultimate theory of weak interactions, the predictions of the model for the interactions of neutrinos with electrons and nucleons have been experimentaliy tested to a level necessary for supernovae theory. There exists, however, no experimental test of the predictions of this standard mudel for neutrino-neutrino scattering. In particular models have been proposed for generating a neutrino Majorana mass in the context of the Weinberg-Salam model as a result of the nonvanishing vacuum expectation value (VEV) of an isotriplet Higgs field. The expected Nambu-Goldstone boson, the Majoron, appears in the model, but its effects are surprisingly benign. The phenomenological implications of these models for terrestrial experiments, for cosmology, and for supernovae (by Dicus et.al) have been studied.

In "Spontaneous Lepton Number Violation and De-Leptonization in Stellar Collapse" (DOE-ER-03992-506) Dr. Dicus, together with E. Kolb and D. Tubbs of LoS Alamos Scientific Lab further analyze the supernova problem, 
paying particular attention to the finite temperature and finite density effects on symmetry breaking. They show that the initial neutrino number in the supernova core prevents symmetry restoration, which might otherwise be expected for the temperatures obtained in stellar collapse and that the breaking of the symmetry results in rapid de-leptonization of the core due to $|\Delta L|=2$ processes. Although the conclusion of core de-leptonization was reached previously (by Dicus et.al) the present mechanism is substantially different from that of prior studies.

The effects of proton decay on the future of the universe has been investigated by Dicus for both open $(k=-1)$ and closed $(k=1)$ Friedmann. universes. This work was reported in previous Progress Reports and has been cited in numerous magazine and newspaper reports as well as in the American Institute of Physics report Physics News in 1982, NOvember 1982. A Scientific American article "The Future of the Universe" (DOE-ER-03992-514) by Dicus, John Letaw of the Naval Research Lab, Vigdor Teplitz of the Arms Control Agency and Doris Teplitz of the University of Maryland was published in the issue of March 1983.

There is a possibility that a magnetic monopole has been observed. The monopole density implied by the observation appears to violate bounds on the density of such particles derived from the total mass density of the universe and from the existence of galactic magnetic fields. Dicus and Teplitz in a Nature article titled. "Circumvention of Parker's Bound on Galactic Magnetic Monopoles" (DOE-ER-03992-515) show that the observation is not inconsistent with these bounds if the monopoles and antimonopoles are bound into positronium - like states with principal quantum $n$ high enough so that the earth's magnetic field will break them apart, but small enough so that the weaker galactic magnetic field will not. They determine a range of values. for $n$, show that lifetimes for such bound states are longer than the current age of the universe and point out that pairs would be sufficiently separated by the earth's field not to enter an experimental apparatus together.

In a recent letter, Wilczek raised the possibility that decay of a heavy neutrino, $\dot{v}_{H}$, into a lighter neutrino, $v_{L}$, might proceed by emission of a familon $f$-- an axion-like particle that connects families of different flavors. He pointed out that familon emission could evade the limits on neutrino masses and radiative coupling constants derived from cosmological 
restrictions on $v_{H} \rightarrow v_{L}+\gamma$. In a paper "Upper Bound on the Decay Constant of Familons" (DOE-ER-03992-519) Dicus and Teplitz point out that, for some $v_{H}$ masses, the $\nu_{H} \nu_{L} f$ coupling constant is restricted by the requirement that primordial $\nu_{H}$. decay occur sufficiently early for $\nu_{L}$ and $f$ to be red shifted enough that their present energy densities not be larger than the cosmological critical energy density. This upper bound on the cosmic energy density of the decay products yields: (1) an upper bound on the familon decay constant, and consequently (2) a lower bound on other $f$ emission rates. By comparing the $k \rightarrow \prod f$ lower bound with the experimental upper bound, we rule out neutrinos that decay by familon emission and have masses between $5 \times 10^{-5}$ and $0.1 \mathrm{MeV}$.

There are two principal motives for interest in pion condensates in neutron matter. The first motive is that neutron star luminosities due to neutrino cooling processes indicate that pions provide the dominant cooling channels for low temperatures. While the observational data is consistent with improved cooling calculations in the absence of pions, the rapid cooling possible in the pion channel might be necessitated by lower limits on the surface temperatures of neutron stars. The second motive is that the pions are charged bosons. Thus a pion condensate might avoid the exclusion energy contributions which charged.leptons and baryons would make to the pressure and energy. Such contributions could seriously affect the bulk, properties of the matter, leading to observable consequences for neturon stars, and perhaps for large nuclei as well. These contributions would be especially drastic if the dominant pion interactions were the attractive ones favoring condensate

- formation. In DOE-ER-03992-516 Professor Gleeson and Dr. Jim Wheeler show that both of these motives can be met by a detailed model using the full spectrum of available hadrons.

The larger phase space accompanying an expanded baryon spectrum allows more efficient cooling in the baryon-baryon channels which presently suffices to explain the observational limits on neutron star surface temperatures. A third feature of a more complete model of hadrons, in particular one based on a constituent model, is a softening of the equation of state brought about by the lowering of the asymptotic relationship between the pressure and the energy. A constituent description of hadrons is asymptotically, free, thus replacing the $P=E$ asymptotic limits expected for any description dominated by massive vector mesons with sufter $P=E / 3$ limits. Thus it 
appears that pion condensates may be unnecessary, and that they rest upon incomplete or mathematically indeterminate bases.

In addition to their density arid binding energy, descriptions of neutron star matter may be usefully characterized by their bulk moduli, and by the spectrum of particles which are included. The obvious significance of the bulk modulus is the support it provides for the neutron star, and its ability to halt the infall of matter in a supernova event. It also provides a connection with the observed "breathing mode" excitations of large nuclei. The spectrum of particles assumes special significance for the nucleonic densities which dominate the composition of massive neutron stars. The overlap of nucleons at these densities provides a strong arguement for a spectrum which includes not only octet baryons, but also quarks. Above nucleon density these quarks should make ever increasing contributions to the equation of state, resulting in an asymptotic equation of state in which the pressure. is one-third of the energy density. Finally, descriptions limited in composition to the first generation of flavor often develop pion condensates above nuclear densities, and it will be interesting to see if these can survive the increased competition provided by the strange hadrons.

In DOE-ER-03992-518 Professor Gleeson and Dr. Wheeler study matter composed of octet baryons, electrons and muons, quarks, nonet scalar and vector mesons, gluons, and the electromagnetic field, as described at the classical level in the background field formalism. Cold equations of state are computed for coupling strengths corresponding to descriptions of infinite nuclear matter with isothermal bulk moduli per nucleon at nuclear density between 25 and $60 \mathrm{MeV}$. Nuclear density was taken to be .19 baryon per $\mathrm{F}^{3}$, with a binding energy or $15.74 \mathrm{MeV}$. Thesc cquations of stat.e are applied to neutron star calculations, and low bulk moduli seem to be excluded. The equation of state for a reasonable bulk modulus is displayed over temperatures between one and one hundred MeV, and adiabatically for entropies per baryon between one tenth and ten $K_{B}$. 
B. Phenomenology of Particle Physics

In the past year, research on the Regge approach to multiparticle dynamics has been very much stimulated by the preparation for an invited talk, which Professor Chiu presented at the 14th International Symposium in Multiparticle Dynamics. The task there was to review the multiparticle dynamics based on the conventional Regge theory. So among other things, his research has been concentrated on the hadron dynamics based on the exchanges and interactions of the Pomeron and the Reggeons.

We have made a systematic study on the data for a large number of processes and found the dominance of the Reggeon-Reggeon-Pomeron vertex contributions in the kinematic region: for the Feynman variable $x>0.8$ and the momentum-transfer-square: $|. t|<1 \mathrm{GeV}^{2}$. We suggested that this region is unsafe for the presently popular models, such as the recombination models and the counting-rule models.

In general, there are now strong experimental indications confirming both the Reggeon-Reggeon-Pomeron and the triple Pomeron predictions. This very conclusion suggests that the general approach based on Reggeons and triple Reggeon interaction is quite relevent and it should eventualily lead to a comprehensive theory for soft-hadron physics.

Also we have turned our attention to models which are aiming at a comprehensive description of the data based on Regge theory. One popular. approach is stemmed from the recognition that the triple Pomeron coupling is smal1. In this context one argues that at presently accessible energies, one may ignore the triple Regge interaction effect and include only the poljypomeron exchanges of the eikonal-model-type. This approach is still very much alive. However, we observed that there are still important corrections for the model, needed to be considered. We are particulariy interested in the effect associated with the proper inclusion of the conservation of energy constraint. Our research in this direction is now in progress:

The other approach is based on the critical Pomeron solution in the Reggeon Field Theory (RFT) of the Pomeron. In this context, we mention our 
results on the multiplicity moment parameter: $\left.C_{p}=\langle\cdot n(n-1)\rangle \ldots(n-p+1)\right\rangle /$ $\langle n\rangle^{P}$. Back in 1975, Canesch $i$ and Jengo and also Suranyi have calculated these parameters for $p=2$ and $p=3$ based on Wilson's $E$-expansion to first order in $\epsilon$. Recently Sanford Wilson and Dr...Chiu extended their calculation for arbitary $p$. The obtained theoretical predictions of: $C_{\vec{p}}$ are in reasonable agreement with the $\bar{p}$ collijer data, at $\sqrt{\mathrm{s}}=540 \mathrm{GeV}$. This agreement between the theory and the data suggests that the scaling behavior of the critical Pomeron solution is indeed lurking precociously. The next step is to check whe ther to higher order in E=expansion, such agreement still persists. Our second order work is now in progress...

How does this Regge approach make contact with presently accepted fundamental theory: the QCD. There are two points which come to mind. "Firstly, through the notion of the dual string, multiperipheral chains in RFT may be closely related to the fluxtube solution in QCD. The latter is believed to be a crucial ingredient for confinement. Secondly, from a general theorem of Grisru and Schnitzer, QCD gluons can be reggeized. So the RFT of the Pomerons we are exploring are expected to share common features with the RFT based on the QCD Pomeron.-- a reggeized gluon in disguise. In this context, we believe that the Regge approach wi11 continue to provide-insights to the eventual QCD description for the soft hadronic processes.

S. Nandi and S:P. Chia-(visitor to CP.T) from the University of Malaya, Kuala Lumpur, Malaysia) investigated the possible low energy effects of a globally supersymmetric SU(3) ${ }_{C} \times S U(2) \times U(1)$ theory. Specifically, they calculated the electric dipole moment of neutron in such a theory (Phys. Lett. 117B, 45 (1982)). To order $g^{4}$, where $g$ is the weak SU(2) coupling constant, they found that the contribution to the intrinsic quark electric dipole moment due to gauge interactions vanishes, as in non-supersymmetric case. When the gluon and gluino radiative corrections were included and supersymmetry was broken, they estimated that the single quark dipole moment could be several orders of magnitude bigger $\left(10^{-28} \mathrm{~cm}\right)$, than the standard SU(3) X SU(2) X U(1) prediction $\left(10^{-34}\right)$. They also examined the importance of penguin diagrams in supersymmetric theories. It was found that the new penguin diagrams introduced by supersymmetry, have a structure similar to usual penguin diagram. Its contribution, though comparable in the case of exact supersymmetry, was shown to be small when supersymmetry was broken (Phys. Rev. D27, 1654 (1983)). 
A wide class of Supersymmetric unified theories have charged fermioni partners $\left(\tilde{W} \pm\right.$ ) Tighter than the $W^{ \pm}$gauge bosons, as neutral partners $(\tilde{Z})$ lighter than the $Z^{0}$. It is of great interest to study the production and decays of these SUSY, particles in the existing and forthcoming colliding beam facilities. S. Nandi, D. A. Dicus, and X. Tata discussed the various decay modes of the $W-$ boson into SUSY particles and also decays of these SUSY particles, in a unified theory in which gravity breaks $S U(2)_{L} \times U(1)$ (DOE-ER-03992-517; Phys. Lett. $B$ in press). They pointed out that if the decay $W$ WZ is kinematically allowed, the subsequent decay of the ganginos will give three charged leptons in the final states, and may provide a definitive signature of supersymmetry.

The recent discovery of the W-boson at the CERN. $\mathrm{pp}$ collider naturaliy leads us to ask whether the $\tilde{W}$ can be seen in the same experiment. S. Nandi, D. A. Dicus, W. W: Repko (Michigan State University) and X. Tata studied the $\tilde{W}$ production the $\overline{p p}$ collider via $W \rightarrow \tilde{W} \tilde{\gamma}(\tilde{\gamma}$ is the photino) and the subsequent decay of the $\tilde{W} \rightarrow e \bar{v} \tilde{Y}$. This will give rise to events with a single electron and no associated charged particles or jets. For electron energies less than $30 \mathrm{GeV}$, the background from direct $W$-decay is sma11. The other sources of electron in this energy range, namely the sequential decays of the form $W \rightarrow \ell v_{\ell} \rightarrow e \bar{v} v_{\ell} \bar{v}_{\ell}$, where $l$ is a heavy lepton or $\tau$, was included. They found that the wino or a new heavy lepton decay provides a substantial excess of single electrons if the wino mass is less than $50 \mathrm{GeV}$ [DOE-ER-03992-521]

The spectrum generating group (SG) approach which has been developed by $A$. Bohm heading an international team of theoreticians is based on very general and well accepted assumptions (Lorentz invariance, SU(3) classification the product form of the interaction Hamiltonian.with $V-A$ and octet transformation properties as well as CVC and the first class condition) but assumes in addition that the flavor group also acts on the masses keeping however, the four velocity operator $\hat{\mathrm{P}}_{\mu}=\mathrm{P}_{\mu} M^{-1}$ invariant. It is this last assumption that predicts the symmetry properties of the magnetic moments in intrinsic magnetons, whereas the conventional assumption is that they are to be taken in proton magnetons. And from this assumption follows also. that the formfactors for the HSD depend upon the internal quantum numbers not only through the Clebsch-Gordan coefficients but also through suppression factors that are functions of the masses.

Numerous fits of the precision values for the HMM have been performed and we found that the SG model predictions are preferred over the conventional 
quark model predictions in proton magnetons. We found that, using only octet currents (Gel1-Mann-Nishijma formula) the agreement is still not good and higher multiplet terms with purely magnetic properties are needed. We have tested two kinds of corrections. U-spin scalar terms which are singled out by the model: independent SG assumptions for the hadron electromagnetic current and octet Uspin vectors, which came from quark mass breaking in a non-relativistic quark mode1. We found that the best fit is obtained for the U-spin scalar current in the SG approach $\left(x^{2}=7.5\right)$ whereas the $U$-spin vector corrections from the broken quark model produce only a very poor fit $\left(x^{2}=64\right)$ :

In our fits of the HSD we have used radiative corrections and $q^{2}-$ dependence of the formfactor which are starting to become important with the improvement of the experimental data. Whereas the conventional fit (Roos fit) makes use of the rates and experimental ${ }^{\mathrm{g}} \mathrm{g} /$ ratios which are extracted from the experimental data under the assumption that all $g_{2}$ are zero. We use correlation coefficients and asymmetries.

New experimental data on HSD have been published ( $J$. Wise et al Phys. Letters 98B, 123 (1981); M. Bourguin et a1 Z. Phys. C12, 307. (1982); P. Keller et al PRL 48, 972 (1982)). Using these experimental data A. Bohm and P. Kielanowski in collaboration with P. Magnollay and A. Garcia made a new fit of the Cabibbo model as we17 as the SG model. They found that the latest value for the rate of $\Sigma^{-} \rightarrow \Lambda \mathrm{e}$, if it does not change, will require small SU(3) correction terms in the current. The new value for $\alpha_{e}^{\sum n}$, however, cannot be explained by any small SU(3) breaking term in the Cabibbo model it cannot be explained by a sizeable second class current, it can only be explained by the SG model and there it does not require SU(3) correction term in the current. If the latest value of the rate for $\Sigma^{-} \rightarrow \Lambda$ ey will change slightly upward, then the SG model will explain all experimental data without a need for SU(3) correction terms in the currents.

In all previous fits of the hyperon decay data of $A$. Bohm et al and other groups CVC was used as an input from which the $f_{2}$ formfactors (and also the $f_{1}$ formfactors) were calculated in terms of the charges and the hyperon magnetic moments: Using the latest experimental results a set of sufficiently accurate HSD data is available to test the models for semileptonic decays separately. It was found that the HSD data alone determine the values $f$ and $f_{2}$ formfactors for the baryons and give values which are very close to the values predicted by CVC. This, therefure, constitutes a test of the CVC hypothesis. 
After this test was performed A. Bohm and collaborators used CVC in order to make a joint fit of all baryon semileptonic decay data and baryon magnetic moments for the SG model as well as for the conventional (Cabibbo and magnetic moments in nuclear magnetors) model. The SG model gives a good fit, the conventional model gives a slightly worse fit if one excludes the new value for $\alpha_{e}^{\sum n}$. But with the new value of $\alpha_{e}^{\sum n}$ the conventional madel is ruled out. This work is contained in DOE-ER-03992-501.

A. Garcia and P. Kielanowski analyzed the possibility of measurement of formfactors from the Dalitz plot (DP) for semileptonic hyperon decays. It was found (Physics Letters 120B (1983) 214) that if the initial hyperons are unpolarized and no final polarization is measured then not all of the formfactors can be determined from the. DP. However, for the polarized unified hyperons (Phys. Lett 121B (1983) 424) this ambiguity is resolved and one can determine formfactors uniquely. In DOE-ER-03992-511 A. Garcia and P. Kielanowski also.. gave several tests of $V-A$ theory. The analysis of $A$. Garcia and $P$. Kielanowski is especially important for the forthcoming experiment for semileptonic hyperon decays that will be performed at Fermilab and may enable a resolution of the existing ambiguities.

Professor Bohm continues to work on the amalgamation of an SO $(4,1)$ gauge theory of extended objects with his earlier de Sitter model of hadrons. This work is carried out in collaboration with Dr. W. Drechsler. Drechsler has developed to So 4,1$)$ gauge theory in the geometrical language of fiber bundles of Cartan taype, and the joint efforts of Bohm and.Drechsler were to unite these two different mathematical descriptions of the same intuitive physical idea. The geometrical language of Drechsler suggests that we expand the concept of space-time beyond its conventional form to include internal coordinates which have a dynamical meaning. It pictures a hadron as an excitation of the de sitter fiber with $10^{-13} \mathrm{~cm}$ radius attached to the points of Minkowski space. In the earlier algebraic and group theoretical language the de Sitter model leads to the relativistic rotator spectrum and the intuitive picture was that hadrons are miniature de sitter spaces of $10^{-13} \mathrm{~cm}$ attached to the space-time points. To obtain the spectrum these $i d e a s$ have to be combined with the spectrumgenerating group approach using infinite dimensional generalizations of the Dirac matrices.

These authors together with N. Mukunda proposed classical models of a relativistic rotator, whcih they called "spinorial models" (Phys. Rev. D22, 1938 (1980)), that overcame several difficulties inherent in the conventional 
theories of classical relativistic rotators.

Starting from these classical models $A$. Bohm and collaborators (R.R. Aldinger, P. Kielanowski, M. Loewe, P. Magnol 1ay, N. Mukunda, W. Drechsler, S.R. Komy, L. C. Biedenharn, :H. van-Dam) in DOE-ER-03992-523 hàve developed a quantum theory for relativistic rotating objects in which the relativistic Hamiltonian is defined by the $(4+1)$ de Sitter constraint given by the second order Casimir operator of the group of motion in the De Sitter fibre with approximate ly $10^{-13}$ on radius. In the first part of this work the quantum observables are defined and the constraint Hamiltonian mechanics is developed. The quantum mechanical equations of motion are obtained following the prescription given by Dirac for the transition from the classical to the quantum level, with the only difference that now the micro-de Sitter Hamiltonian is is used. This leads to a helical motion of the particle position around a center of mass position ("Zitterbewegung") .. which was excluded in most previous relativistic rotator models by the additional Pryce-constraint that lead to the identification of the particle position and center of mass position; and the identification of spin and intrinsic angular momentum. The de Sitter Hamiltonian also leads to a mass versus spin spectrum which is consistent with experimental hadron spectra and characteristic of a relativistic rotator.

In the second part of this work (A. Bohm, M. Loewe, L.C. Biedenharn, H. van Dam Phys Rev $D$ to appear) the simplest representation spaces of this de sitter model. for the quantum relativistic rotator are obtained. It is shown that its representation. spaces reduce into a discrete direct sum of representation spaces of the Poincare group which establishes the fact that hadrons (described by irreducible representation of the Poincare group) are different mass spin levels of the quantum relativistic rotator.

In a series of experiments pion-proton bremsstrahlung $\pi^{ \pm} p \rightarrow \pi^{ \pm} p \gamma$, has been measured in the region of the $\Delta$ resonance. In principle, comparison of this data with calculated cross sections could yiel.d.values for the $\Delta^{++}$ and $\Delta^{0}$ magnetic movements. Unfortunately these calculations are not unambiguous because; in order to see the effects of the magnetic moments, the photon energy must be too large to allow accurate calculation with the Low theorem. Thus the data has been analyzed using a particular method which ignores the width of the $\Delta$. Dicus, a student Henry Schreiner, and Teplitz (DOE-ER-03992-500) have investigated the effect of the finite width. They 
find the analysis of the $\pi^{+} p$ data to be ambiguous. Including the effects of the non-zero $\Delta$ width seems to mean that the present data does not uniformly give a solution although, for those cases where a solution is found, it agrees well with the SU(6) or bag model values. Analysis of the $\pi^{-p}$ data is less ambiguous but was not preformed before. We find $-17.1 \leq \mu_{\Delta}^{0} \leq 9.6$ in units: of eh/MC which is consistent with the SU(6). (or bag mode1) result of zero.

In a recent Phys. Rev. Letter it was argued that the effective potential for the Higgs scalars, with the one loop radiative correction included, gave an upper bound on the mass of the Higgs boson. Dicus and Gleeson in a paper published in. Phys. Rev. Letters (DOE-ER-03992-524) show that this is not true by explicitly finding additional. solutions to the equations for the Higgs mass. 
C. Quantum Field Theory, Supersymmetry and Models of Particles

Professor Sudarshan in colliaboration with Balachandran, Marmo, Mukunda, Ni.lsson and Zaccaria have been carrying out a systematic study of monopole configurations in grand unified theories. They have constructed a fibre bundle formalism very well suited for practical calculations. They have discovered that in monopole sectors with nonabelian magnetic flux there are topological obstructions to a global definition of some of the "unbroken" transformations. In particular for the symmetry breaking chain $S U(5) \rightarrow S U(3) \otimes S U(2) \otimes U(1)$ locally even though color is a local symmetry the full color group cannot be globally defined and thus monopoles break color. A preliminary publication has been made in Phys. Rev. Letters $\underline{50}, 1553$ (1983) but more complete presentations are in course of publication. They have also studied the motion of test particles in the field of monopoles; they find that the transformations of the "unbroken" group cannot all be globally implemented as canonical transformations. For the $S U(5) \rightarrow S U(3) \times U(1)$ model color multiplets are not globally defined while for $S U(5) \rightarrow S U(3) \times S U(2) \times U(1)$ both color and electroweak multiplets cannot be defined globally. Where are several distinct subgroups $K_{T}$, $K_{T}$, which can be globally implemented.

It is well known that if a symmetry is spontaneously broken, the generator of the corresponding symmetry transformation does not exist in that its action on the (translationally invariant) vacuum state yields a state of infinite norm. For the spontaneous breaking of a global supersymmetry, if one regards the supersymmetry algebra as a statement on the operators, one is led to conclude that a necessary and sufficient condition for a spontaneous breakdown of supersymmetry is that the vacuum expectation value of the Hami1tonian be positive. (Admittedly, this value is infinite, being the integral of a constant over all space-time). In a Poincare invariant theory, however, a non-vanishing vacuum expectation value of the Hamiltonian is a signal for the spontaneous breakdown of Lorentz invariance. This would lead one to believe that supersymmetry could not be spontaneously broken without a simultaneous breakdown of Lorentz invariance.

This, coupled with the fact that various models exist in the literature in which supersymmetry is spontaneously broken, led Professor Sudarshan and Dr. Tata to look for a structure in which the spontaneous breakdown of supersymmetry does not signal that of Poincare invariance. Working in collab- 
oration with Professor Joseph Schechter of Syracuse University, they have shown that supersymmetry can be spontaneously broken without any violation of Poincare Invariance if the supersymmetry algebra is understood to be an equality between the brackets of the group generators with the dynamical variables (the vield operators). Then, the breaking of supersymmetry has nothing to do with the spectrum of the Hamiltonian. They have also pointed out that this "weakening" of the algebra does not alter any conclusions that have been obtained about supersymmetric Lagrangian quantum field theories. This work has been published in Physics Letter $B$.

Dr. Tata and Professor Sudarshan have constructed a fermionic Lee model for which the spectrum can be computed exactly and the eigenvectors constructed recursively. This paper has now been published (J. Math. Phys. 24, 1810 (1983)) and is one example of an exactly soluble field theory in 3 dimensions.

Until recently, it was generally believed that supersymmetry (SUSY) is always broken by. finite temperature effects. This conclusion is based on the fact that finite temperature Green functions do not satisfy the SUSY Ward identities, they obey at $T=0$. This, in turn leads to the inequality of the boson and fermion masses for $T \neq 0$. The basic reason for these results is the difference in the thermal distribution for bosons and fermions. In a recent paper (Nucl. Phys. B207, 15 (1982) Van Hove has argued that finite emperature effects do not necessarily violate susy Ward identities if the thermal averaging is done with care. To explain this, we recall that for any symmetry, a Ward identity may be obtained by equating the ground state expectation value of the variation under the symmetry transformation of a function of field operators to zero, i.e.

$$
\left.<\delta_{0}{ }_{0} \equiv<[\epsilon 0,0]\right\rangle_{0}=0
$$

Here, 0 is any field operator, $Q$ is the generator of the symmetry transformation (and by definition, annihilates the ground state) and $\epsilon$ is the parameter of the transformation. At $T=0$, the ground state is the Fock vacuum, whereas for $T \neq 0$ it is a mixture defined by the cariunical density operator $\mathrm{A}=\mathrm{e}^{-6 \mathrm{H}}$. For the case that $Q$ is a generator of SUSY $\epsilon$ is a Grassman variable. Van Hove has pointed out that $<|\epsilon O|>= \pm \epsilon<|0|>$ according as the state vector $1>$ refers to a bosonic (fermionic) state. This is crucial for studying the 
supersymmetry of a system in a thermal background since the ground state involves a weighted average over states containing an arbitrary number of bosons and fermions. For the case of a two-level quantum system, Van Hove went on to explicitly illustrate that the SUSY Ward identities continue to be true even for $T \neq 0$.

This led Professor Dicus and Dr. Tata to investigate the corresponding situation for a fully relativistic supersymmetric field theory. Following Van Hove, they showed that at finite temperature, the SUSY Ward identities led to relations between graded thermal averages of field operators rather than ordinary thermal averages. Thus, it became clear why the usual thermal Green functions did not satisfy the SUSY Ward identities and further why the inequality of the boson and fermion masses did not imply any breaking of SUSY. Dicus and Tata went on to explicitly verify the SUSY relations between the graded Green functions were indeed satisfied. Their analys is is applicable. for a wide class of theories and to all orders in perturbation theory. They conclude, therefore, that finite temperature effects in themselves do not necessarily violate SUSY. They have pointed out that in SUSY theories, with an internal symmetry, non-perturbative effects that cause the phase transition that results in the restoration of an internal. symmetry can cause a breaking of SUSY. They have also noted that since the response of a thermodynamic system to external perturbations is expressed in terms of the ordinary (ungraded) Green functions, the SUSY relations between the graded Green functions may not have a physical significance.

Professors Chiu and Sudarshan, in collaboration with B. Misra of the Solvay Institute (Brussels) have reinvestigated the possible implication of quantum zeno paradox and its implications for. proton decah; contrary to the results of L. A. Khalfin, they find no appreciable suppression of proton decay.

If one of the $\tilde{W}^{\prime} s$ ( $\tilde{Z}^{\prime} s$ ) have mass less than the mass of the $W(Z)$ (as is the case in a wide class of SUSY theories), then, they will be produced in the electron-positron colliding beam facilities to be buitt at SLC and LEP. In the currently popular $S U(2)_{L} \times U(1)$ supergravity gut models, masses and coupling of the most of the SUSY fermions and scalar quarks and leptons are determined in terms of two parameters, which could be taken as to be $M_{W}$ and Mg (gravitino mass). S. Nandi, D.A. Dicus, W.W. Repko and X. Tata calculated the total electron positron annihilation cross-section into all the allowed SUSY particles, upto each beam energy of $80 \mathrm{GeV}$. (Univ. of Texas preprint 
DOE-ER-03992-522, 1983). For a wide range of masses of the Wino and gravitino, they find that theis cross-section is much larger than that expected from the usual quarks and leptons (even including a new family of quark and sequential. heavy lepton). Thus, a measurement of total annihilation cross-section at SLC or LEP will be sufficient to demonstrate the existence or non-existence of SUSY. They also calculated the total width of $Z$ (including the contributions due to decays into various supersymmetric channels) and found that for small wino mass $(\sim 20 \mathrm{GeV})$, the width could be larger than the usual non-supersymmetric prediction by as much as $50 \%$. Thus a measurement of the width could provide a separate signature for SUSY.

In the past several years considerable progress has been made in the lattice gauge theory, especially in the realm of understanding nonperturbative phenomena within the theory. In the context of Monte Carlo calculation, we now have numerical evidence for confinement in SU(2), SU(3) gauge theories, chiral symmetry breaking in QCD and some rough but encouraging meson and baryon mass calculations. To optimists in this approach there is a hope that in the near future more precise numerical calculations of the properties of QCD and the related gauge theories will be accomplished and the strong interactions will be "understood".

While computor calculations are very necessary, we believe that at this stage it is also important to further explore the approximate technique to complement the Monte Carlo investigation. Recently Bitar et al have demonstrated the approximate correctness of the Midgal recursion in the context of SU(2) lattice gauge theory. More specifically they found that their phase diagram for the fundamental-adjoint mixed action is similar to that given by the Monte Carlo calculation. Intrigued by its simplicity and its potential usefulness, Steve Eubank and Dr. Chiu have been exploring the ramifications of the Migdal recursion relation. Here are some of their results. They found that the positivity condition defined by Osterwalder and Seiler in the construction of the lattice gauge theory imposes an important set of constraints on the Migdal recursion relation. Also within the framework of the recursion relation; they have discovered a method to extract the string tension of the theory. Based on their method, they have evidence that the asymptotic free scaling parameter does depend sensitively on the mixing angle for the case of the mixed action. This is in agreement with an earlier observation made by Mutter and Schilling using the Monte Carlo approach. Their eventual objective 
is to investigate the feasibility of the calculating hadronic mass ratios in the context of the Migdal recursion relation.

Within the mean field approximation, the vacuum structure of the matter fields in gauge theories on lattice have been studied in various contexts. For instance, it has been studied in a U(1) lattice gauge theory with scalars, and in nonabelian gauge theories with fermions, etc. Among various conclusions, it has been shown that in the strong coupling domain, chiral symmetry would always be spontaneously broken. This has led to a conjecture by Quinn, Drell. and Gupta that the composite models of quarks and leptons should not be a nonabelian-gauge theory of the usual type. We shall refer to. this as the no-go senario for the composite models of quarks and leptons.

Following an earlier suggestion by Quinn, to elucidate the corresponding situation for composite models involving both fermions and scalars, Pisin Chen and Dr. Chiu have considered a color SO(N) theory, in which fermions and scalars are assigned to the fundamental representation. This theory has the usual dimensionless gauge coupling $g$ and $\mathrm{a} \lambda\left(\phi \phi^{+}\right)^{2}$ interaction term. Similar to the approach of previous authors, based on the $1 / \mathrm{g}^{2}$ expansion, we studied the vacuum structure in the strong coupling domain.

They found that to zeroth order in $1 / g^{2}$, within a certain range for the parameter $\lambda$, those mixed color states which contain both the non-colorsinglet bosonic components and the corresponding color-conjugate fermionic components should necessarily have their energy eigenvalues higher than that of the lowest possible value in the color singlet state without nonsinglet components. Then they proceeded to investigate the situation to next order in $1 / \mathrm{g}^{2}$. Within the same range of the parameter values, the singlet ground state is now contaminated with mixed states having nonsinglet components. However, the mised states are suppressed by $1 / \mathrm{g}^{2}$. Furthermore, they alter the ground state energy only to order of $1 / \mathrm{g}^{4}$. We recall that the earlier arguement on the breaking of the chiral symmetry by Quinn et al is $1 / g^{2}$ effect. So this earlier arguement is still applicable for the present case. This then lead them to propose the extended no-go senario for theories, where fundamental scalars are present in the composite models for quarks and leptons.

Professor Bohm continues to work on the amalgamation of an $\mathrm{SO}(4,1)$ gauge theory of extended ohjects with his earlier de Sitter model of hadrons. 
This work is carried out in collaboration with Dr. W. Drechsler. Drechsler has developed to so $(4,1)$ gauge theory in the geometrical language of fiber bunders of Cartan taype, and the joint efforts of Bohm and Drechsler are to unite these two different mathematical descriptions of the same intuitive physical idea.

Bohm and Gadella have continued the investigation of Gadella on the Rigged Hilbert Space formulation of ideal unstable particles. Starting by constructing a rigged Hilbert space of Hardy class functions restricted to the positive half of the real live, they can describe resonances as well as virtual states (poles of the S-matrix on the negative real axis of the second sheet) as generalized eigenvectors of the total Hamiltonian. They define time evolution on the functionals for the case when the testfunction space is invariant under the adjoint time development operator. This requirement splits the time evolution group into two semigroups, one for $t>0$ and the other for $t<0$, which can be separately extended to two timedevelopment semigroups of operators, hence displaying the irreversibility. of the decay - and formation processes.

In DOE-ER-03992-526 Professor Ne'eman reviewed the status of GUTS, with the present raised bound for the proton lifetime $\left(>10^{31}\right.$ years, excluding "simple" SU(5)). He particularly looked at cosmological applications and discussed the new Inflationary Model. This will be published as "Prospects for Further Unification", in E. Teller 75 th Anniversary Festschrift. 
D. Geometric Formulations and Algebraic Models

Following the geometric derivations last year of the ghost-antighost symmetrized Curci-Ferrari BRS-like algebra Professor $\mathrm{Ne}^{\prime}$ eman has further refined the definition of products of ghost-fields in the pathintegral formalism. In a paper with J. Thierry-Mieg (DOE-ER-03992-513) to be published in the 1982 Jerusalem International Conference on Differential Geometrical Methods in Physics he has constructed a formally unitary Lagrangian model for an internal supersymmetry by gauging the even solvable Lie algebra appearing as the even part of the product of the simple superalgebra with the Grassmann Manifold of forms over space-time. The construction explains such features as matter-field ghosts, the correlation of chirality with the superalgebra's representations' gradings, the Kronecker metric and the vector ghosts in the adjoint representation.

Professor $\mathrm{Ne}$ 'eman presented at the 2nd Shelter Island Conference (June 1983) the method of gauging an internal supergroup through the use of the natural Grassmann algebra of forms over space-time as a basis for the ring of parameters in the exponentiation of the supergroup (Proc. Nat. Acad. Sci., USA $\underline{79}, 7068-7072$ (1982)). He shows that this method is an example of a generalized Cartan-integrable system. This is given in detait in DOE-ER-03992-498 where a formally unitary Lagrangian model gauging an internal supersymmetry is proposed. The even subalgebra is gauged as a Yang-Mills theory, while the odd generators are gauged - according to Freedman's method - by skew tensor fields, equivalent dynamically to scalar Higgs fields. Chiral Fermions are incorporated following Townsend's construction, and form irreducible supermultiplets graded by their helicity. The method will be further discussed at the XII International Symposium on Group Theoretical Methods in Physics, Trieste (1983). In a paper (DOE-ER-03992-499) honoring Marcos Moshinsky on his sixtieth birthday, Professor Ne'eman analyzed the algebraic systems of the string. Dual Models or Strings (hopefully generalizable to Membranes and Lurips) have played an important role and raised many hopes between 1968 and 1974, when they suddenly receded into the background as a result of the success of Yang-Mills gauge theories (Quantum "Chromodynamics, "QCD"). There is at present an active comeback, partly as a result of attempts to prove the equivalence between Yang-Mills gauges and Strings, in the search for a demonstration of Quark Confinement features in 
OCD. Since Strings confine quarks almost by definition (quarks are the endpoints of the open String), equivalence would thereby imply confinement in $Q C D$, as yet unproven except in the lattice approximation. The string formalism also appears to have applications outside of the Physics of Particles and Fields, wherever there is a need to compute sums over random surfaces, such as in the 3-dimensional Ising model. This is because just as pointparticles generate paths, and path integrals are taken over all possible paths, Strings generate world-sheets, and one integrates over all possible sheets. Strings also bear a strong relationship to Gravity and to Supergravity in several different--büt perhaps interconnected--ways.. The as yet speculative generalization to Membranes and Lumps may be important, with the latter bearing a direct relationship to Affine Gravity.

In DOE-ER-03992-512, pubiished in Foundations of Physics, Professor $\mathrm{Ne}$ 'eman reviewed the mathematical theory of $S L(n, R)$. and its double-covering group $\overline{S L}(n, R)$, especially for $n=2,3,4$. He discussed a variety of physical applications, showed that $\dot{S L}(3, R)$ provides holonomic curved space ("world") spinors with an infinite number of components, and, as an example constructed the relevant holononic "manifield" for the gravitational interaction of a proton.

Professor Ne'eman has noticed that modern developments in Theoretical Physics may throw new light on some of the more difficult probiems in the interpretation of Quantum Mechanics and may provide unexpected solutions. He has shown that the non-local features of Quantum Mechanics of the EinsteinPodolsky-Rosen type exist semi-classically in a Yang Mills gauge theory (DOE-ER-03992-525). In these theories, it is the gauge connection that ensures parallel transport, and correlates events at finite distances." This may hint at the need for a modified geometrical structure for ordinary Quantum Mechanics, considering that other non-local quantum effects (such as the Aharonov-Bohm effect) have already been shown to originate in the topology.

While in. Europe the past year. Dr..A. Stern -(in collaboration with Drs. A.P. Balachandrain. (Syracuse), G. Marmo (Naples) and B.-S. Skagarstam (Goteborg)) studied topics concerned with the general subject of gauge theories and fibre bundles (to be published in "Lectures in Physics", Springer-Verlag). The topics concerned the dynamics of point particles; eg. particles in interaction with magnetic monopoles, spinning particles and their coupling to electromagnetism and gravity, interactions with a non-Abelian gauge field, and 
Kaluza-Klein descriptions of point particles. There does exist a certain amount of literature written by general relativists and particle physicists which attempts to explain the language and techniques of fibre bundles. Gen-. erally in these reviews, the concepts of fibre bundles are illustrated by field theoretic examples like the gravitational and Yang-Mills systems. This practice tends to create the impression that the subtelties of fibre bundles can be understood only through the medium of complicated field theories. This impression is however, false because simple systems with gauge invariance exist in plentiful quantities as indicated in the above examples. The examples thus serve as an introduction to the study of fibre bundles as well as having an intrinsic physical. interest in their own right. In addition the fibre bundle language was found useful for the above systems, in that they provided for a singularity-free Lagrangian description. Some further topics which were examined were: (1) the extension of the above systems to particles with fermionic degrees of freedom, (2) the quantization of the above examples and (3) the general question of how global Lagrangian descriptions can be constructed from systems which adnit only local Lagrangians. (2) was nontrivial because the dynamics of the examples given were defined on group manifolds.

In collaboration with Dr. B.-S. Skagerstam, Dr. Stern examined a curious relationship between topology and spontaneous symmetry breaking in supersymmetric quantum mechanics, (to be published). More specifically they examined some supersymmetric particle systems coupled to an external field which admits topological soliton solutions. Zero mode solutions indicating that supersymmetry is unbroken in the quantum theory, are found to exist only for certain values of the topological index. Examples of the above were found in 1, 2 and 3 dimensions corresponding couplings to kinks, vortices and tt Hooft-Poljrakov monopoles.

Prof. A.P. Balachandran, Drs. V.P. Nair, S.G. Rajeev and A. Stern have reexamined the topological solutions found by T.H.R. Skyrme to the chiral model. They found the soliton states to be long-lived with exotic quantum numbers. It was speculated that these states may be related to the recently observed anomalous nuclei. Prof. E. Witten has later proposed that these topological solitons could actually represent the usual baryon states. 
APPENDIX A

TITLES OF DOE REPORTS

SINCE DOE-ER-03992- 496 
TITLES OF DOE REPORTS SINCE DOE-ER-03992-496

DOE-ER-03992-496

The University of Texas at Austin Progress Report "Research Program in Elementary Particle Theory" by E.C.G. Sudarshan and Y. Ne'eman

DOE-ER-03992-497 The University of Texas at Austin Research Proposal "Research Program in Elementary Particle Theory" by E.C.G. Sudarshan and Y. Ne!eman

DOE-ER-03992-498 Exterior Gauging of an Internal Supersymmetry and SU(2/1) Quantum Asthenodynamics, by $Y$. Ne'eman and J. Thierry-Mieg

DOE-ER-03992-499 Algebraic Insights on Quantized Strings, Membranes and Lumps, by $Y$. Ne'eman

DOE-ER-03992-500 Magnetic Moments of $\Delta^{++}$and $\Delta^{0}$, by D.A. Dicus, $H$. Schreiner and V. Teplitz

DOE-ER-03992-501 Fits Combining Hyperon Semileptonic Decays, Magnetic Moments and the Conserved-Vector-Current Hypothesis, by $A$. Bohm and $P$. Kielanowski

DOE-ER-03992-502 Monopole Topology and the Problem of Color, by A.P. Balachandran, G. Marmo, N. Mukunda, J. Nilsson, E.C.G. Sudarshan and F. Zaccaria

DOE-ER-03992-503 Paraxial Wave Optics and Relativistic Front Description I: The Scalar Theory, by E.C.G. Sudarshan, R. Simon and N. Mukunda

DOE-ER-03992-504 Paraxial Wave 0ptics and Relativistic Front Description II: The Vector Theory, by N. Mukunda, R. Simon and E.C.G. Sudarshan

DOE-ER-03992-505 Spontaneously Broken Supersymmetry and Poincare Invariance, by Xerxes Tata, E.C.G. Sudarshan and J. Schecter

DOE-ER-03992-506 Spontaneous Lepton Number Violation and De-Leptonization in Stellar Collapse, by D. A. Dicus, E. W. Kolb and D. L. Tubbs

DOE-ER-03992-507 On Purturbation Theory at Finite Temperature, by D. A. Dicus, D. Down and E. W. Kolb

DOE-ER-03992-508 Supersymmetric Field Theories at Finite Temperature, by $D$. A. Dicus and X. Tata

DOE-ER-03992-509 Symmetry Breaking in the Spectrum Generating Group and Its Experimental. Tests, by P. Kielanowski and P. Magnollay

DOE-ER-03992-510 Examples of Group Contraction by A. Bohm and R. Aldinger 
$\begin{aligned} \text { DOE-ER-03992-511 } & \begin{array}{l}\text { Two Theorems on the Unique Determination of Form Factors } \\ \text { in Polarized Hyperon Semileptonic Decays, by A. Garcia } \\ \text { and P. Kielanowski }\end{array}\end{aligned}$

DOE-ER-03992-512 Some Double-valued Representations of the Linear Groups by $Y$. Ne' emán

DOE-ER-03992-513 A Lagrangian for SU(2/1) Quantum Asthenodynamics, by J. Thierry-Mieg and $Y$. Ne'eman

DOE-ER-03992-514 The Future of the Universe, by D. Dicus, J. Letaw, D. Teplitz and V: Teplitz

DOE-ER-03992-515 Possible Circumvention of Parker's Bound on Falactic Magnetic Monopoles, by D. Dicus and V. Teplitz

DOE-ER-03992-516 DO Pions Condense in Neutron Star Matter by J. W. Wheeler and A. I1. Gleeson

DOE-ER-03992-517 W Decay in Süergrävity GUTS, by D. Dicus,'s. Nandi and $X$. Tata

DOE-ER-03992-518. Neutron Star Matter at Finite Temperature, by J. Wheeler and $A$. Gleeson

DOE-ER-03992-519 Upper Bound on the Decay of Familons, by D. Dicus and V. Teplitz

DOE-ER-03992-520 Heavy-mass power counting theorem and a proof of decoupling in the momentum subtraction scheme, by T. Schimert and C. C. Chiang

DOE-ER-03992-521 Electron Spectra from Wino Decay at the pp Collider, by.D. Dicus, S. Nandi, $M$. Repko and $X$. Tata

DOE-ER-03992-522 Electron-Positron Annihilation Cross-Section in $\mathrm{SU}(2)_{L} \times U(1)$ Supergravity, by D. Dicus, S. Nandi, W. Repko, and $x$. Tata

DOE-ER-03992-523 The Relativistic Rotator I. Quantum Observables and Constrained Hamiltonian Mechanics, by R.R. Aldinger, A. Bohm, P. Kielanowski, M. Loewe, P. Magnollay, N. Mukunda, W. Drechsler, and S.R. Komy

The Relativistic Rotator II. The Simplest Representation Spaces, by A. Bohm, M. Lowew, L.C. Biedenharn and H. van Dam

DOE-ER-03992-524 Comment on Upper Bounds on the Higgs Boson Mass and the Coupling Constant, by D. Dicus and A. Gleeson

DOE-ER-03992-525 Classical Geometric Resolution of the Einstein-PodolskyRosen Paradox, by $Y$. Ne'eman

DOE-ER-03992-526 Prospects for Further Unification, by Y. Ne'eman 
DOE-ER-03992-527 Triple" Reggeon Interactions and Reggeon Dynamics in High Energy Collisions, by Charles B. Chiu

DOE-ER-03992-528 Decay Phenomena and Rigged Hilbert Spaces: Two Lectures, by $A$. Bohm and M. Gadella

DOE-ER-03992-529 Fourier Optics for the Maxwell Field: Formalism and Applications, by N: Mukunda, R. Simon and E.C.G. Sudarshan

DOE-ER-03992-530 Generalized Rays in First Order Optics: Transformation Properties, by R. Simon, E.C.G. Sudarshan and N. Mukunda

DOE-ER-03992-531 Realization of First Order Optical Systems Using Thin Lenses, by E.C.G. Sudarshan, N. Mukunda and R. Simon 


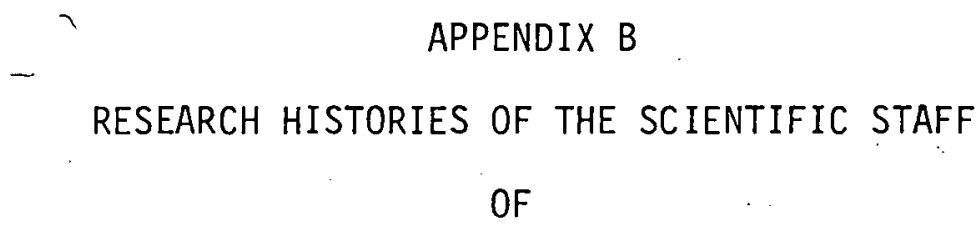

THE CENTER FOR PARTICLE THEORY 
RESEARCH HISTORY

\section{Yuval Ne'eman - Professor of Physics and Co-Director, Center for Particle Theory}

Yuval Ne'eman has combined several careers besides the one in Physics. The circumstances of Israel's emergence into Statehood in the midst of a struggle for survival made him dedicate to purely military activities, the first twelve years after getting his degree in Engineering. He achieved stature as one of Israel's top thinkers in the domains of Strategy and Defense Policy.

His scientific career was initiated in 1961, working in London (Imperial Co.17ege). where he had made the transition in 1958-61 and obtained a Ph.D. from London University. Here he conceived and published his theory of Unitary Symmetry, which can now be considered as the foundation of most developments in Particle Physics in the last two decades. Returning to Israel he conceived the idea of a fundamental sub-particle triplet, with the observed nucleons and hyperons being constituted of various combinations of three such basic units. This idea, further developed by M..Ge11Mann and G. Zweig (with the units christened as "quarks") is now the key to our further dynamical understanding of the hadrons.

Ne'eman pursued his research in Israel first at the Atomic Energy Commission Laboratories, where he became Scientific Director, and then as a Professor of Physics at Tel-Aviv University, where he founded the Physics Department. He Iater became. University President (1971), leaving the Presidency after four years, in order to serve as Senior Advisor to the Minister of Defense (acting as Deputy to the Minister in all Defense Policy) yet again in combination with the position of Chief Defense Scientist. . He resigned from these positions after a year, on a matter of National Policy, and held no Government or University responsibilities from 1976 to 1981, when he was elected to the Israefi legislature. In: 1982 he joined the Cabinet as Minister of Science and Development. Since 1968, (and except for the year 1975), Ne'eman has held a half-time position as Professor of Physics at the University of Texas, Austin, where he founded the Center for Particle Theory of which he is now a Co-Director. This arrangement (similar to one made by the University of Texas at the same period with. Prof. I. Prigogine, with respect to the Center for Statistical Mechanics) was part of the University's drive for excellence, which brought about the creation of several such research Centers. 
Ne'eman has also spent several years as a Guest Professor (or Fairchild Distinguished Fellow) at Cal Tech, and has been a guest for shorter periods at IAS Princeton, at SUNY Stony Brook, the IHES Paris and CERN.

Returning to Ne'eman's research contributions, it was in a 1962 paper of his with three of his students that the first clear cut demonstration was given of the experimental good fit of Unitary Symmetry in Strong reactions, in which the previously favored "Sakata model" had failed. Later on, in 1964 he suggested "the Fifth Interaction" that the SU(3)-breaking effect observed in the mass spectrum was due to an interaction with a relatively long lived vector-meson resembling the $\phi$. It should be noted that the $\psi-J$ particle discovered in 1974 fits most of the characteristics predicted in that paper (even the mass, though the reasons quited for such a mass in the paper were irrelevant in the final result). For the leptons, a reordering in their systematics in connection with the Weak Interaction (1962) was later used by A. Salam in developing his (1968) version of the Gauge Theory of Weak and Electromagnetic Interactions. A 1964 article with M. Gell-Mann proved that the algebra of Weak and Electromagnetic Charges is isomorphic to a doubled SU(3) (or to "Chiral" Unitary Spin). This was the algebra used in all the 1965-68 work on "Current-Algebra". With $\mathrm{A}$. Joseph, Ne'eman classified in 1969 all representations of the algebra of local surrent densities.

In the further development of the quark model, Ne'eman identified in 1966 (together with N. Cabibbo and L. P. Horwitz) the algebraic pattern behind all high energy quark-model results, a doubling of Unitary Symmetry which preserves in fact the Unitary spins of Quarks and of Antiquarks separately in these regimes. Note that the currently popular Zweig-Itzuka-0kubo rule, used to explain the slow decays of $\phi, \psi$ and of the recentily discovered $T$, are part of this algebra, which also predicts the famous Levin-Frankfurt $\sigma(\pi N) / \sigma(N N) \rightarrow 2 / 3$, etc.. In a series of articles between 1967 and 1974 Ne'eman succeeded in providing this phenomenological algebra with a deeper theoretical foundation, based on an extension of the ideas of bilocal current operators and the null-plane expansion. This explanation can now be considered "definitive", as a logarithmic approximation of the presently favored $Q C D$, for instance. 
Another line of work dealing with the Strong Interactions, but with many applications in other fields, was initiated by Ne'eman in 1965 in two articles with M. Gell-Mann and Y. Dothan. This pointed out the role (till then unnoticed) of non-compact groups which reproduce the set of all solutions of certain problems in Quantum Mechanics in one single representation (the idea was suggested independently by E. C. G. Sudarshan and by A. Barut and A. Bohm). This approach, followed in 1966 by an application to S-matrix singularities, was later much in use in the development of Dual Models by $G$. Veneziano and others, who have also applied extensively another 1966 idea of Ne'eman's (with N. Cabibbo, L. Horwitz and J. J. J. Kokkedee). The suggestion was that high energy phenomena were dominated by a "Pomeranchuk trajectory" whose intercept differed from one by a 'smal1 number (the direct experimental prediction of diminishing cross sections failed, but the idea is now applied to the "unrenormalized" trajectory)

One particular suggestion of the 1965 Dothan-Gell-Mann-Ne'eman paper had dealt with $S L(3 R)$ as a model for the hadron excitation bands represented by Regge trajectories. With D. W. Joseph, Ne'eman showed in 1970 the existence of double-valued spinor representations of that group. In 1976, the subject acquired new dimensions: the existence of these bandspinors provided for intrinsic shear currents, thus going beyond the 1965 Dothan-Gel1-Mann-Ne'eman representations, which corresponded to time-derivatives of Gravitational Quadrupoles (an idea which was tested in deformed Nuclei by L. C. Biedenharn and collaborators in 1972). With F. Hehl and E. C. Lord, Ne'eman now showed that such shear, together with scaling and spin densities would constitute the intrinsic hypermomentum tensor of General Relativity. In the metric affine extension of G. R., the shear-scale-spin tensor is coupled to the connection, in addition to the coupling of the energy-momentum to the metric. In an intriguing phenomenological observation, $\mathrm{Ne}^{\prime}$ eman noted that it was just these three currents which appeared to be approximately conserved in the quark model: spin-independence (SU(6)), scaling and the pattern of Regge bands. However, since $S L(3 R)$ is the algebra of volume preserving deformations, this may just correspond to confinement.

This recent work has led Ne'eman to a discovery in General Relativity: the existence of (infinite) Spinor representations of the Group of 
General Coordinate Transformations ("bandors"). Almost any textbook in GR has stated that the G.C.T.G. has no spinor representations, so that this was a real innovation and may yield many applications (there is already one important mathematical application by B. Kostant).

In other work in Nuclear Physics and Astrophysics, Ne'eman suggested in 1968 the existence of hypercollapsed nuclear isomers. The idea was independently suggested by A. Bodmer in 1970 and by T.D. Lee (in the context of Boson condensation) in 1972.

In General Relativity, Ne'eman invented White Holes ("Lagging Cores") to explain quasars in 1965, and developed the idea in 1967 and 1972.

The discovery of CP (or 1) vrolation led Ne.'eman (1969-1974) to a Statistical Mechanics study of the possible interrelation of the microscopic arrow of time with the thermodynamical one and. With Cosmology. Surne of thal. work was crucial in the 1978-1980 work of Yoshimura and others in applying Grand Unified Theories to cosmology.

Since 1974, the discovery of Supersymmetry has led Ne'eman to the development (with S. Sternberg) of the Mathematical theory of Graded Lie Algebras. With M. Gell-Mann he presented the Group Structure of Supergravity (including the special role of the $N=8$ extension) independently of $i$ ts discovery in field theory by D. A. Freedman and collaborators and S. Deser and B. Zumino. With T. Regge, he has developed a Geometric Theory of Gauges, for Groups acting on Space-Time. The theory reproduces General Relativity and Supergravity but also provides new insights about possible modifications or generalizations. In 1980, Ne'eman showed that $\mathrm{SU}(\mathrm{N} / \mathrm{I})$ was a symmetry of the $\mathrm{N}$ - extended supergravity.

Since 1977, Ne'eman has worked on a reformulation of the theory of Gravity. The aim is to develop a version that would be fully renormalizable. It could be very different from Einstein's at the quantum level, provided its macroscopic limit is close enough to General Relativity. With F:W. Hehl, J. Nitsch and $P$. Von der Heyde, Ne'eman published in 1978 a first version of such a theory, the "Poincare Gauge Theory" (PGT). It has since been shown that it indeed has a "teleparallelism" limit which can only be distinguished from GR at the 5 th ppN level. Beyond the above-mentioned indications of a possible link between Metric Affine Gravity and Quark confinement, the PGT 
appears to contain a "strong" confining term directly. With Dj. Sijacki, Ne'eman has meanwhile further developed the understanding of $\overline{\mathrm{SL}}(4, R)$ or $\overline{\mathrm{GL}}(4, R)$ band-spinors, and has outlined what an Affine theory of Gravity should look like. Ne'eman also used these band-spinors to construct two and three dimensional analogs of the 1 -dimensional spinning-string superalgebras of Ramond and Neveu-Schwarz.

With the discovery of a third generation of quarks and leptons, $\mathrm{Ne}$ 'eman called for the abandonment of the idea of an ordinary simple group to provide the generation structure and also for a view in. which quarks and leptons are themselves composite objects. The idea has generated some interesting models, especially one by Harari and Shupe. As a result, G. 't Hooft has suggested compositeness equations.

In 1979, Ne'eman invented a new type of gauge theory, using a supergroup internaliy. The resulting supersymmetry is a symmetry of the quantum Lagrangian, rotating fields into:ghosts. The supergroup SU(2/1) provides a: perfect embedding of the Weinberg-Salam (SU(2) XU(L)) in a simple group, therefore highly constraining all free parameters and many otherwise arbitrary choices. With S. Sternberg, he has extended this group in 1980 to $\mathrm{SU}(5+\mathrm{k} / 1)$, to include color. It predicts a set of $2^{k+1}$ identical "copies" (or generations.) of the 15 basic quarks and leptons. The SU(7/1) represents an Anomaly-free SU(7) $X U(1)$ renormalizable gauge theory, constrained by the supergroup. It predicts 8 generations and imposes "critical" Quantum Chromodynamics (QCD) i.e. 16 "flavors". This is unique in reproducing a factorizable Pomeranchuk trajectory in High Energy Scattering. Recently, Ne'eman and J. Thierry-Mieg have provided a dynamical version, manifestly unitary apparently - though final proof requires additional work.

With J. Thierry-Mieg, Ne'eman has identified the geometric role of the renormalization "Faddeev-Popov". ghosts, thus providing a further advance in the imposition of Unitarity. He has since shown that this applies to noninternal gauges such as gravity as wel1. A symmetrical extended BRS algebra provides geometrical identification for the "antighost" as well as the "ghost" fields. This version can be adapted to supergroups such as $\operatorname{SU}(2 / 1)$ or $\operatorname{SU}(7 / 1)$ as well.

Ne'eman is a Foreign Associate of the U.S. National Academy of Sciences and of several other Academies. He has published ten books and 
about 250 publications in Scientific Journals. He is the recepient of numerous prizes and awards, including the Einstein Meda1.(1970) and the Wigner Medal (1982). (see Physics Today 36, \#2, pp. 51-52 and 85; a1so Springer Verlag Lecture Notes in Physics 180 , M. Serdaroglu and E. Inouu eds., Proc. Istanbu1 Col1. on Group Th. Methods in Physics 1982, pp. 566-569. 
RESEARCH HISTORY

E. C. G. Sudarshan - Professor of Physics and Co-Director, Center for Particle Theory

E. C. G. Sudarshan got his B.Sc. (Honors) and M.A. Degrees in Physics at the University of Madras and his Ph.D. in Physics at the University of Rochester. His interests and research contributions cover a broad sweep of physics.but all along Particle Theory has has held the center stage. From an early stage in his career as a physicist he has generously given of his time and energy in assisting, directing, instructing and counseling numerous physicists many of whom have gone on to make a name for themselves. He considers himself sometimes as a teacher, sometimes as a research worker, but always as a scientist.

He started his research career with the study of multiple scattering in nuclear emulsions at the Tata Institute of Fundamental Research. His interest in more fundamental problems of what has now come to be known as Particle Physics started here and came into its own during the two years he spent as a graduate student at the University of Rochester. His doctoral work led to the discovery of the now classic V-A interaction. He formulated the first statistical theory of antinucleon annihilation and introduced the Lorentz-invariant phase space density for statistical theory of meson production. The first application of broken symmetry in particle physics, the equal spacing rule for magnetic moments of isospin multiplets was derived by Marshak, Okubo and Sudarshan during this period. This application naturally led to Okubo's work on-unitary symmetry and the Ge11-Mann-0kubo mass formula.

Pruressur Sudarshan spent the two years 19b/-1959 as a Research Fellow at Harvard University with Professor Julian Schwinger. Inspired by Schwinger he studied quantum theory of higher spin fields and demonstrated a general theorem on the existence of secondary constraints and consequent problems of quantization of higher spin fields with special reference to the inconsistency of spin $3 / 2$ fields in an external electromagnetic field. This work, which was completed in collaboration with Kenneth Johnson, has been the impetus for a whole new area dealing with relativistic wave equations, particularly the work of Velo and Zwanziger on the acausality of higher spin field propogation; and of Arthur Wightman and his various associates on the problem of wave equations 
in external fields.

A different area of research concerned itself with analytic properties of field theoretic amplitudes. In collaboration with walter Gilbert and Stanley Deser he developed a new family of integral representations which have come to be called the DGS representations.

The work on weak interactions continued with the increasing confirmation of the universal V-A theory. He collaborated with the Rochester group in elucidating many of the subtle properties of weak interactions, in particular, the SU(2) properties of weak currents. Among them are the first announced connection between the axial vector hadronic current and the pion source, the first characterization of the $I=\frac{1}{2}$ current rule for strangeness changing decays and the consequences of $\Delta I=\frac{1}{2}$ for $K^{0}$ decay.

Sudarshan returned to Rochester as an Assistant Professor on the University faculty in 1959 and was promoted to the rank of Associate Professor in 1961. During this period, he moved into new territory. Anticipating the great interest in Lie groups, he formulated classical mechanics in terms of Lie groups; this interest has now completed its promise in a new book on Classical Dynamics written in collaboration with Narasimhiengar Mukunda and published by John Wiley in 1974. In collaboration with two students, Thomas Jordan and Douglas Currie, he investigated relativistic Hamiltonian theories and proved a fundamental no-go theorem which has come to be called the No Interaction Theorem. He laid the plans for a systematic study of irreversible quantum processes considered as stochastic dynamics of quantum mechanical systems. This work has been continued, in collaboration with T. Jordan, P. M. Mathews and, particularly, Vittorio Gorini, and has received the attention of mathematicians who call them positive and completely positive maps.

The study of particle physics was continued during this period. Robert Marshak and George Sudarshan published a thin volume of the Theory of Elementary Particles, which documented the insights of the late fifties and early sixties. Along with Susumu Okubo and Robert Marshak he found the general solution to a class of integral equations in the course of a study of lambda hyperon deday: this solution is now known in the literature as the Omnes' Solution. Unitary symmetry came to be discovered during this time and 0kubo developed his celebrated mass formula as a more substantial application of 
broken symmetry. During this time, Sudarshan and his co-workers developed a general theory of sum rules using the so-called Smushkevich method; these were some of the earliest derivations of consequences of unitary symmetry. Particularly notable are the results on electromagnetic moments and mass differences.

During this period, the most important new undertakings were the formulation of two new concepts: that of quantum theory with indefinite metric and the theory of tachyons. While Dirac had pointed out the possibility of an indefinite metric and Pauli had reviewed it, the first serious work on this was by Suraj Gupta in quantum electrodynamics. Sudarshan took up this work but showed how it could be applied to remove divergences from local field theory and yet retain the conventional probability interpretation of quantum mechanics. Along with Arons and Han he applied it to quantum electrodynamics. The developments in the area were reviewed in his report to the Solvay Conference in 1968. Since that time, the subject has received wide attention due to the eloquent advocacy of T. D. Lee. In their papers Lee and Wick raised the possibility of use of complex bare masses: this turns out to be an excellent idea though the manner in which they implemented their program led to violation of Lorentz invariance. This difficulty was pointed out by Sudarshan and co-workers.

The idea that particles may travel with speeds exceeding that of light in a relativistic theory, which Sudarshan proposed in the fifties, was met with strong criticism and it was published only when it was rewritten subsequently in collaboration with Bilaniuk and Deshpande. Many authors have now taken up the theme; tachyons along with magnetic monopoles and quarks and conspicuous by their absence on the scene.

During his Rochester years, Sudarshan contributed to quantum field theory, particularly in reference to the possible existence of multiple vacuum states within the Wightman framework. His work with Bardakci showing the trivial S-matrix for a scalar field theory with a polynomial source predates the work of Borchers. His work on parastatistics in collaboration with Ryan is one of the clearest derivations. But the most important contribution to quantum field theory was to be quantum theory of optical coherence in which he showed that there is a complete equivalence between the semiclassical theory 
of partial coherence and the results of quantum electrodynamics. In collaboration with Leonard Mandel and Emil Wolf, he also gave a formulation of photoelectric counting distribution. The fundamental insights gained in this area are summarized in the book QUANTUM OPTICS, coauthored with John K! auder, published in 1968.

In 1964-1965 Sudarshan was Guest Professor at the Institute of Exact Sciences, University of Bern; he also made guest visits to Brandeis University and the Institute of Mathematical Sciences in Madras. During this period, he showed in collaboration with O'Raifeartaigh and Santhanam and with Leutwyler, that if certain consequences of symmetry (the Smushkevich type sum rules) hold, then a symmetry follows. He also showed that this was related to, but distinct from, the Cutkosky mechanism of induction of symmetries. The principle is closely related to the algebra of currents, and used as such by Cook, Goebel and Sukita and by Kuriyan and Sudarshan for strong coupling theories. During this time, the question of combining internal symmetry and the Lorentz group was triggered by MCGTinn. Sudarshan proved two basic no-go results of this type of questions and introduced Lochlainn 0'Raifeartaigh to the problem which he subsequently solved completely.

In 1965 Sudarshan joined Syracuse University as Professor of Physics and helped create an active, though small, high energy theory group there. The work on higher symmetries was now taken up in earnest. During this time, Lochlainn 0'Raifeartaigh discovered his theorem and Moo-Young Han did his work on the Han-Nambu model. Noninvariance groups were discovered during this time by Sudarshan along with Mukunda and 0'Raifeartaigh. Jacob Kuriyan, Narasimheingar Mukunda and George Sudarshan did some pioneering work on the theory of unitary representations of noncompact groups, which has come to be known as master analytic representations. Relativistic SU(6), the orbital excitation model of SU(6) $\times 0$ (3) were discovered during this time. So was the theory of Primary Interactions, which gave a natural basis for vector dominance models.

But his most important contribution during this time was to current algebras. Krishnan Raman and George Sudarshan generalized the Ward-Takahashi method to the case of nonabelian symmetries and derived the generalized WardTakahashi Identities from which follow a host of results including the AdlerWeisberger relations and the Tomozawa-Weinberg-Raman scattering length rela- 
tions. This basic result and its application to pion-nucleon scattering are published in Physics Letters and the Physical Review.

In 1968 Sudarshan spent a semester as a Visiting Professor at the Centre for Advanced Research in Physics and Astrophysics at Delhi University. During this period, he formulated a theory of tachyon fields incorporating the reinterpretation principle in collaboration with Jawharlal Dhar; and a review of superluminal physics along with 01exa-Myron Bilaniuk. The celebrated theorem of Pauli on the connection, between spin and statistics presupposes the restriction to finite component relativistic fields. Sudarshan reexamined this question and showed that it is possible to derive the relation between spin and statistics from the symmetry between emission and absorption. He showed, in two papers presented to the Nobel Symposium and to the Indian Academy of Sciences, that ultimately the connection between spin and statistics is traceable to the rotation group in three dimensions for which the fundamental bilinear form is symmetric for tensor fields and antisymmetric for spinor fields.

During this period, Sudarshan got seriously interested in philosophy, particularly, the nature of causality, time, coherence, perspective and cognition. These concerns and the fruits of this study have strongly influenced him in his understanding and presentation of physics. It is curious that both tachyons and quantum theory with indefinite metric raise questions in these philosophical domains.

The University of Texas at Austin invited him to become Professor of Physics in 1969 and to assume the post of Co-Director of the Center for Particle Theory in 1970. In association with Yuval Ne'eman he founded a strong group. of particle theorists at Austin of which he continues to be a member. Together with Relativity and. Astronomy there is an exciting and active group now which has the honor of having John Wheeler and Ilya Prigogine.

During these years, Sudarshan has continued his work in particle physics. Along with co-workers, he analyzed high energy scattering data for tachyonic resonances, studied the analytic properties of transition amplitudes in indefinite metric theories and the question of violation of causality in such theories. The study of quantum theory of fields with spin. $3 / 2$ was taken up in collaboration with Bruce Mainland; they showed that not only the 
Heisenberg equations of motion but the entire Poincare Lie algebra is properly realized by the field.

The problem of action-at-a-distance in relativistic theories was reinvestigated. Since he had proved the no-interaction theorem in local relativistic theories, this was only just! He showed that every action-ata-distance theory can be embedded in a local field theory in a natural manner; and that any finite theory of this kind automatically implied an indefinite metric. A general theory of physically meaningful indefinite metric theory was the natural follow-up and this work has been carried out as the theory of "shadow states". Shadows are states that are involved in formulating the dynamics but not involved in the unttarity sum.

logether with J. P. Hsu and two students, Allen Underwood and Enrique Mac, Sudarshan studied unified gauge theories and gave an alternate formalism using Lagrange multiplier fields; and applied it to construct a number of models. In most cases this method gives rise to different and, often, simpler Lagrangians, but essentially the same S-matrix as given by the usual theory.

Description of higher spin particles continues to be a problem and relativistic wave equations are actively being studied. William Hurley and George Sudarshan have studied a general class of such equations giving unique mass and unique spin. A new class of "barnacled wave equations" have been introduced and studied. Aslam Khalil has completed a doctoral thesis on generalizations of this scheme and related topics.

Multiple particle production at high energies is an active field of interest. In collaboration with Charles Chiu and $K$. Wang, he has formulated. a hydrodynamical theory of multiparticle production with frame-independence.

Dynamical maps have been investigated in collaboration with Vittorio Gorini. The extremal maps of a two-level system have been obta'ined. Like finding the unitary representations of a noncompact group, this is a "first". For completely positive maps the use of Choi's theorem enables one to find all the extremal elements in a simpler manner. In collaboration with Drs. V. Gorini and A. Frigerio of the University of Milan and Dr. A. Kossakowski of the University of Torun he has completed a review article on quantum Markovian equations. 
Particle physics concepts have application to other fields and several such applications have been carried out. The competition between species cán be modeled by coupled fields and one can thus generate Hamiltonian models of "interaction" between biological species. This work is done in collaboration with S. N. Biswas. The temporal behavior of a repeatedly observed quantum system is a fascinating topic and certain features of this were investigated in collaboration with Professors Charles Chiu. and Baidyanath Misra. Misra and Sudarshan have aiready shown that continuous observation, followed in quantum mechanics, leads to a Zeno's paradox. This has naturaliy led to a closer study of unstable systems and their time evolution and the results are presented in a paper by Misra, Chiu and Sudarshan. Sudarshan has also shown how it introduces an interaction between a classical and a quantum system and thus provides a basis for measurement theory by embedding the classical system in a quantum system with continuous super-. selection rules. Sudarshan and Mukunda have shown that Nambu dynamics is essentially Hamiltonian dynamics, seen in a new perspective.

The problem of measurement in quantum theory has been the subject of discussion for many decades, but this was always done without a premise statement of the interaction between a quantum system and the classical measuring apparatus. Sudarshan has recently succeeded in discovering an embedding of classical dynamics in a quantum dynamics with continuing superselection rules. Several papers on this has been published in collaboration with B. Misra, S. Gautam and T. Sherry.

The study of time evolution of unstable systems has served as a trigger to crystaliize some of Sudarshan's long standing interest in the analylic continuation of the state space of quantum theory. A general theory is formulated for analytic functionals as the framework of such a theory. Work on this topic is continuing, though two papers have-already been completed in collaboration with V. Gorini and G. Parravicini.

The physics of consciousness has always fascinated Sudarshan, both in its metaphysical and empirical aspects. The research work done at MERU in Switzerland, discussions with Professor G. Globus at the University of California at Irvine and Professor D. Shainberg of New York University, as well as Professor B. Josephson at Cambridge have contributed to a focusing of this interest. Eontinuing discussions with Professors John Wheeler and Ilya Prigogine 
and their guests at the University of Texas, including Professors Stapp, Minsky, Scully, Peres, and d'Espagnat on measurement theory and artificial intelligence and further discussions with $\mathrm{Dr}$. H. Mathews at the University of Texas serve to elucidate and delimit the general problem. This work is still in its infancy:

Quarks are enigmatic entities and perhaps they do not obey ordinary statistics. Sudarshan has continued his study of parastatistics in collaboration with Mukunda and Mehta; and of finding completeness theorems for coherent states for parabose fields. He has also found a new family of coherent states in terms of which the density matrix of an illumination field can be expressed as a weighted sum of projections. A related completed work is the pursuit of light rays in wave optics including quantum electrodynamics and radiative transfer.

The space-time development of physical processes is of particular interest to Sudarshan. He has pursued a study of it in hadron dynamics and quantum optics. The quantum Zeno's paradox can be made to manifest itself in successive collisions inside a nucleus. P. Valanju, C. Chiu and Sudarshan have developed the theory in a paper recently completed. The application of these ideas to the possible modification for the equation of state of matter at very high densities as obtains in the interior of stars have been studied in collaboration with P. Valanju and C. C. Chiang.

The unification of bosons and fermions into a single multiplet is possible in the framework of supersymmetry which extends the usual symmetry formalism to accommodate a graded Lie algebra with spinor generators. Along with Jue Changkeun and C. C. Chiang, Sudarshan has studied superfields with interna 1 symmetry in a series of three papers which clarffies much of the existing literature as well as provide for the consideration of higher spin equations.

The relationship of the symmetry of the equations of motion (automorphisms) and the symmetry of the realized physical theory (internal automorphisms) has been studied extensively in recent years with particular reference to spontaneous and dynamical breakdown. In collaboration with Balachandran, Mukunda, Nilsson and Zaccaria, Sudarshan has discovered a new mechanism of reduction of the internal automorphisms of a system and illus- 
trated it for a $\mathrm{GL}(3, \mathrm{R})$ model. The $\mathrm{GL}(3, \mathrm{R})$ automorphism group carried by a triplet of pairs of variables automatically implies the internal automorphisms to be reduced to a 3-parameter subgroup and one pair of canonical variables.

The interaction of relativistic particles with local generators of the Poincare group in which the particle trajectories are world lines has been the subject of study by Currie, Jordan and Mukunda two decades ago when they proved the fundamental no-interaction theorem. Sudarshan and Mukunda have recentiy restudied this problem with particular reference to Dirac's constraint dynamics. They are able to show how to construct two classes of such theories, but it is essential to extend Dirac's ten parameter Lie algebra into an eleven-parameter Lie algebra; this work also elucidates the failure of earlier attempts to find invariant world lines within the ten generator formalism of Dirac. They have extended the work with a critical examination of the underlying postulates in collaboration with Goldberg.

In collaboration with Balachandran, Mukunda, Nilsson and Zaccaria, Sudarshan has investigated the conditions under which one can pass from the Hamiltonian system to an action principle in a global manner. This problem is reduced to the context in which the closed 2-form of the Hamiltonian formalism is also exact. In these cases where it is not exact one can show the equivalence to path space dynamics which can be reduced to a classical fiber bundle. These authors have also generalized relativistic dynamics of particles using the constraint formalism on extended group manifolds.

Grand unified theories continue the direction of unification beginning with the $V-A$ theory which unified hadronic and leptonic weak interactions; and the standard unified electroweak SU(2) $\times U(1)$ theory. One consequence of. these theories is that the Weinberg angle which is arbitrary in the standard theory gets definitively fixed in these GUT theories. Sudarshan and collaborators have investigated the problem and shows that the fixing of the Weinberg angle depends only on the grand multiplet structure and not on the GUT group.

In grand unified theories great interest attaches to the predictions for proton decay; it follows that if one goes beyond SU(5) and SO(10) single family theories to more general family unification schemes the groups have to more general, and hence new diagonal interfamily gauge bosons, may exist. 
Nandi, Stern and Sudarshan have shown that in $S O(n)$ models no such diagonal gauge bosons can exist. In the direction of grander unification is the possibility of combining gravitation with all other interactions. Ward and Sudarshan have recognized the close structural similarity between spin properties of Dirac particles and the SO(10) unification of GUT. They have used this similarity to construct a fully covariant theory involving gravitation as well as strong, electromagnetic and weak interactions.

Informal component relativistic equations are known to be appropriate for describing composite systems. Recently Dirac has discovered an equation for describing such composite systems which has only positive energy timęlike solutions but it had the defect of not being able to incorporate electromagnetic interactions. Sudarshan, Mukunda and Chaing have been able to remedy this defect.

The problem of composite structures which act as if they are elementary has been of considerable interest. In this context the equivalence of direct four-particle interactions versus interactions mediated by a Yukawa quantum. has been studied. Tata, Sudarshan, Chiu and Chiang have demonstrated that the strong coupling transmutation of the mediated interaction model to the direct interaction model obtains if and only if certain infinite. energy contributions are removed, thus resolving long standing ambiguities in the field. This result is demonstrated by a direct solution of many particle sectors.

Zeno mass particles are of special interest in models nf 117 tra high energy physics. Those of higher.spin are particularly interesting since the matrix elements of local operators between one-particle states have different properties for spacelike and timelike momentum transfers. Sudarshan has investigated the possibility of eliminating charged massless higher spin particles on this basis.

A11 grand unified theories exhibit monopole solutions with non-trivial topological properties. In collaboration with Balachandran, Marmo, Mukunda, Nilsson and Zaccaria, Sudarshan has studied the classical mechanics of a particle in just such a monopole field. They find that in the field of a monopole with a non-Abelian magnetic flux there are topological obstructions to a global definition of some unbroken symmetry transformations. These results suggest that in many grand unified theories the color symmetry or the electroweak symmetry is broken in a rather novel fashion. 
Sudarshan is a Fellow of the Indian National Science Academy and a Fellow of the American Physical Society. He is the author of three books, one on elementary particle physics, one on quantum optics and one on classical mechanics. Sudarshan has also pursued the close identity of spirit between Indian philosophical thought and twentieth centure physics, particularly modern particle physics... This work has not so far been published in research papers. 
RESEARCH HISTORY

Arno Bohm - Professor of Physics

As a student at the Freie Universitat Berlin in 1962, Arno Bohm worked on axiomatic quantum field theory. He applied the axiomatic field theory of Bogojubov, Medvedev and Polivanov to quantum electrodynamics and showed that the asymptotic form of the currents agrees with the expressions of the renormalized theory. This was later published in a paper with Polivanov. (Nuov. Cim. 40, 1110 (1965)).

During the period 1963-1964, Arno Bohm was an instructor at the Institute of Technoloq.y in Karlsruhe, where he worked on problems of clcmentary particle phenomenology (calculation of pion-nurlenn scattering phase shifts).

In 1964 as a Research Associate at the University of Marburg, Arno Bohm started work on group theoretical methods in particle physics and continued this work with A. 0. Barut at the International Centre for Theoretical Physics in Trieste, where A. Bohm was an IAEA Research Fellow, 1964-1966.

During this time, he suggested (together with A. 0. Barut, Phys. Rev. 139B, 1107) using groups not only as symmetry groups but to describe all states of a physical system by an irreducible representation of a group ("Dynamical group"). The same suggestion was later made by Mukunda, 0'Raifeartaigh, Sudarshan ("non-invariance group") and Dothan, Gell-Mann, Ne'eman, who coined the presently accepted name of "spectrum generating group" for it.

During the same time, he also worked on the mathematical methods of quantum mechanics. He made the suggestion to formulate quantum mechanics in the Rigged Hilbert Space with algebras of continuous operators and thus give a mathematically rigorous presentation of the Dirac formalism in quantum mechanics. (A similar suggestion was made independently by J. E. Roberts).

While $A$. Bohm was a research associate at Syracuse University, during 1966-1968, he gave the construction of the Rigged Hiluerl Space for representations of Lie groups and the proof that the space of differentiable vectors for semi-simple Lie groups is a nuclear space. (J. Math. Phys. 8, 1551 (1967)). (The same construction was suggested later by B. Nage1). His major effort during this period was devoted to working on his suggestion to use the connection between the desitter group and Poincare group for the problem of the 
mass spectrum of elementary particles. This led to the introduction of an elementary length of $1 / 3 \cdot 10^{-13} \mathrm{~cm}$ and the idea that hadrons are miniature desitter spaces of infinite time and finite $\left(1 / 3 \cdot 10^{-13} \mathrm{~cm}\right)$ space extension. (Years later the same picture for hadrons was also obtained by differential geometrical arguments using fibre bundles by W. Drechsler, Fortsch. Phys. 23 (1975).

A Bohm continued the work on the desitter model, when he came in 1968 as an associate professor to the University of Texas. In 1969 a representation of the full relativistic symmetry (a combination of the Poincare algebra, C, P, T and an infinite dimensional generalization of the Dirac $\gamma-$. matrices) was introduced, which was called Generalized Dirac Representation, because it describes baryons and mesons in the same way as the space of the solution of the Dirac equation describes the electron (Phys. Rev. D3, 367). This was combined with the above desitter model for the description of the hadron spectrum (Phys. Rev. D3, 377; Phys. Rev. Letters 23, 436).

In 1970-1971 A. Bohm extended his idea of a quantum mechanical description (i.e., by an algebra of operators) of particle properties to include the algebra of charges. This was in a certain way a continuation of the work he had done previously together with E. C. G. Sudarshan on the semi-leptonic and leptonic decay of hadrons. In the spring of 1971, while A. Bohm was visiting scientists at the Max-Planck Institute in Munich, it was shown that the resulting algebraic model has many structures in common with the algebra of currents. During 1977-1972, this algebra of transition operators was applied to a unified description of the radiative and leptonic decay of vector mesons, partially in collaboration with J. Nilsson at the Institute for Theoretical Physics in Goteborg.

As a continuation of this program, A. Bohm suggested in 1974 to use a minimal set of assumptions: 1) Poincare invariance and C, P, T properties; 2) SU(3) as a spectrum generating group; 3) V-A for the operators that describe the transitions (currents) to calculate weak leptonic and electromagnetic decay properties. Work has been done in three areas of this research program. Together with M. Igarashi and J. Werle (University of Warsaw) the weak leptonic decay of pseudoscalar mesons investigated. Together with A. Garcia (Mexico), J. S. Nilsson (Sweden) and R. B. Teese, the hyperon leptonic 
decays were studied and an improvement of the Cabibbo model was suggested. Together with R. B. Teese and graduate students at Texas this program was applied to the calculation of electromagnetic decays of vector mesons in the framework of SU(4).

This resulted in two discoveries: First that - as expected - the explanation of the decays $V \rightarrow e \bar{e}$ and $V \rightarrow P_{\gamma}$ required in addition to the Clebsch Gordan coefficients a suppression factor of the same kind used for the weak decays; and second, that the Gel1-Mann-Nishijima formula for the electromagnetic current operator is good for the $V \rightarrow$ ee decays but may be insufficient for the radiative decays. This led to the postulation of a purely magnetic SU(3) scalar term in the electromagnetic current operator, the necessity of which has recently been confirmed by the Rochester experiment of $\rho \rightarrow \pi \gamma$. The resulting theoretical scheme then allowed to fit all existing radiative decay rates. An application of this scheme to the Hyperon Magnetic Moments, predicted their symmetry properties in intrinsic magnetons. whereas in the conventional models the magnetic moments are taken in proton. magnetons.

In connection with the writing of a text and monograph on Quantum Mechanics the Rigged Hilbert Space formulation of Quantum Mechanics was again taken up. It was suggested that decaying states be described by generalized eigenvectors of an essentially selfadjoint Hamiltonian with complex eigenvalues and a Breit-Wigner energy distribution (Gamow vectors), an idea which had also been independently proposed in a different form by Baumgartel and which has presently become quite popular. In a review paper that stresses the advantages of the Rigged Hilbert space formulation of quantum mechanics it was shown that these Gamow vectors are the unifying entities of S-matrix poles, quasistationary states and resonance phenomena.

Another old idea that has been taken up again is to consider hadrons as micro-desitter spaces attached to the space-time points and to derive a mass spectrum in this connection. This idea has reappeared as a gauge theory of strong interaction with $\mathrm{SO}(4,1)$ as the gauge group and has been combined with the dynamical stability group idea using $\mathrm{SO}(3,2)$ as the spectrum generating group. The minimal coupling has been generalized to an operator form which relates the Poincare group to a desitter group and gives a spectrum that compares well with the experimental data. 
During an Inter-Academy exchange visit in Moscow a collaboration has been started with $V$. Shelest, A. Sukhanov and others on a book which has been given the working title "Quantum Physics of Atoms, Nuclei and Elementary Particles" and which is to appear as a revised version of the book "Quantum Mechanics".

A. Bohm gave a series of lectures at the Summer Institute in Turkey Ju7y-August 1979 on Gauge Groups and Spectrum Generating Algebras. In November, 1979 he also visited various research institutes in the Soviet - Union within the US-USSR Academy Exchange Program and gave a number of lectures on the Rigged Hilbert space formulation of decaying states, on Spectrum Generating $S U(n)$ and its application to radiative decays and on Spectrum Generating Algebras and SO(4,1) gauge groups. 


\section{RESEARCH HISTORY}

\section{Charles B. Chiu - Professor of Physics}

Dr. Chiu completed his Ph.D. thesis work in high energy experimental physics, at the University of Ca'lifornia at Berkeley in 1965. His thesis is on "Pion-nucleon charge exchange angular distribution, from 500 to $1300 \mathrm{MeV}$." This work provided additional constraints to the determination of pion-nucleon phase shifts. After this work, he joined the theoretical group at the Lawrence Radiation Laboratory in Berkeley. This marked a transition in his research career. Since then, he has been working on theoretical physics problems. During his stay in the theoretical group (1965-1967), he worked on different aspects of the Regge pole model. He was one of the first authors who recognized the experimental confirmation on the "nonsense-wrong-signature zero" in the Regge pole theory. During this period, through several collaborative papers, he worked on the classification of sense-nonsense choosing mechanisms in the Regge pole theory and the different Regge pole solutions for pion-nucleon and nucleon- nucleon scattering.

For the period 1967 through 1968, Dr. Chiu worked in the theory division at CERN as a visiting scientist. During this period, he and Dr. J. Finkelstein proposed and developed the "hybrid model" for hadron two-body scattering at small as well as large momentum transfer regions. This model is similar to the "Weak cut model". He and Dr. J. Finkelstein also derived the $\phi-\omega$ ideal mixing from the exchange degeneracy condition. During this period, he and Dr. A. Kotanski clarified the connection between the Regge pole phase factor and the "Schmid loop".

For the period 1968 to 1969, Dr. Chiu worked in the Cavendish Laboratory; Cambridge, England, as a senior research fellow. He continued to investigate the large momentum transfer scattering phenomena. He delivered a review paper on nonforward scattering in the Theoretical Physics Meeting at Rutherford Laboratory in December, 1968. An extended version of this work was later published in the Reviews of Modern Physics. During this period, Dr. Chiu collaborated with Dr. R. J. Eden and Mr. M. B. Green. They developed the phase contour model which provides a general framework for the description of the phase of hadron amplitude satisfying the general properties: crossing, analyticity, unitarity and Regge asymptotic behavior, etc. 
From 1969 to 1971, Dr. Chiu worked at Ca7tech. From 1969 to 1970, he was a research fellow, and from 1970 to 1971, the Richard Case Tolman Senior Research Fellow there. During these two years, he studied the high energy collision phenomena from the point of view of Regge poles and cuts. He made a systematic survey on the strong points and the weak points of the Regge pole model, and also the inadequacy of cut models. A summary of this survey was presented at the American Physical Society meeting, November, 1970, sponsored by the CPT group here. An extended version of this work was later published in the Nuclear Physics. During his stay at Caltech, he was also a co-organizer of the Caltech conference on high energy phenomenology, and a co-editor of the conference proceedings, in which the need for a phenomenological approach to the strong interaction physics is stressed. Through collaborative work, Dr. Chiu investigated the factorization property of the dual resonance propagator and derived the "generalized ward identity" in the dual resonance model. He and Dr. Robert Heinman formulated the statistical bootstrap model with spins, and demonstrated the relation between the angular momentum spectrum of the dual resonance model and that of the statistical bootstrap model.

Dr. Chiu joined the Department of Physics at the University of Texas at Austin in 1971 as an Assistant Professor. During 1971-1972, he continued to work on the systematics of two-body scattering phenomena at high energy. Based on the statistical bootstrap model, he proposed a "strong central absorption prescription" for two-body scattering, which qualitatively accounts for the successes and the failures of the Regge pole model. He contributed a review article on the "Evidence of Regge "Pole Model in Hadron Collisions" published in Annual Review of Nuclear Sciences, 1972. He reformulated the statistical bootstrap model, found analytic solutions, and gave new physical interpretation to the model. Influenced by local research interest on shadow state theory, with Dr. C. C. Chiang, he also worked on the mass renormalization problem based on the dispersion relation and the Feynman diagram techniques.

During 1972-1973, Dr. Chlu's main research interest was directed toward the formulation of a bootstrap model which accounts for hadron interaction dynamics. An input to the bootstrap model involves the multiparticle production physics, as well as two-body scattering physics, with the unitarity 
relation playing a crucial role linking the two-body scattering amplitude to 2 to $\mathrm{n}$ amplitudes. As a first step toward this goal, he began to explore various phenomenological models for multiparticle productions. Collaborating with Dr. K. H. Wang, he developed an independent cluster emission model for multiparticle production. Collaborating with Dr. R. Gleiser, Dr. Chiu further investigated the ramification of his modified statistical bootstrap mode1.

During 1973-1974, with his general interest in multiparticle production models, he was intrigued by the rise of the total cross section in the ISR region. During this year, he worked on the application of the absorption to the popular models of multiparticle productions: the multiperipheral model and the independent emission model. He and Dr. K. H. Wang and, at an carly stagc also joined by Dr. R. Gleiser, developed the absorption formalism for multiparticle production and achieved to demonstrate that the "absorbed independent cluster emission model" provides a unified description to the elastic data including the rise of the total cross section, the inclusive data and the multiplicity distribution data. This, to our knowledge is the first phenomenological model which simultaneously accounts for such varieties of data. During this period, Dr. Chiu, together with his student E. Ugaz, found an empirical "fixed-power-extrapolation scheme" for the single particle inclusive distribution in the fragmentation region in high energy multiparticle productions. A similar behavior is also found for a hypothetical example of the dual resonance model. With this fragmentation distribution within the framework of the Regge-Mueller model the inclusive rapidity-distribution is specified at all energies. Based on the independent cluster emission model, the specified inclusive distribution leads one to predict the energy dependence of the average multiplicities, and of a temporary rise of the total cross section and the multiplicity distribution. They are all in reasonable agreement with the data.

During 1974-1975, his main research was on the investigation of hydrodynamical models for multiparticle production in high energy hadron collisions. He views hydrodynamical models as providing a general statistical approach to final state interactions among multiparticles, which are produced within a small space-time volume. He, together with E. C. G. Sudarshan and K. H. Wang, proposed a new hydrodynamical model -- the frame independence symmetry model 
for multiparticle production. In contrast to Landau's model, where one assumes there is initially a global thermal equilibrium, within this new proposal the initial thermal equilibrium of matter is reached only at the local level. At high energies, Landau's version violates the uncertainty principle, but this new proposal does not suffer the same criticism. Further on, he and $K$. H. Wang investigated the pion momentum distribution at $90^{\circ}$ in pp collision based on this new model and the notion of evaporation. With the parameters of their model completely specified from a fit to the data at $1500 \mathrm{GeV}$. incident laboratory energy, predictions at other energies ranging from 25 to $1500 \mathrm{GeV}$ show a characteristic anti-shrinking energy dependence and are in good agreement with the data. They have also investigated several technical points of hydrodynamics: e.g., the hydrodynamical model with massless constituents and the constraints imposed on a hydrodynamical system when it undergoes a conical expansion.

During 1975-1976, Dr. Chiu continued his research in the cluster production model. He and K. H. Wang further developed the model applying to the charge transfer distribution, two-particle correlation function, and the rapidity-gap distributions. They concluded that the data require a certain characteristic correlation effect within a cluster for a wide range of cluster decay models. In a separate investigation he, together with $\mathrm{Dr}$. D. Tow, found that if one assumes some $N \bar{N}$ cluster production, the observed rise of the single pion inclusive cross section in the central region at ISR energies may be correlated to the corresponding rise in the $\bar{p}$ inclusive cross section. Along the line of strong interaction dynamics, he, together with $\mathrm{Dr}$. D. Tow and Mr. M. Hossain, formulated a simple SU(3) breaking scheme for the dual bootstrap model. The soluliurs found are in reasonable agreement with the cross section and the multiplicity data. Stimulated by the work of Prof. R. Logan and his collaborators on the statistical bootstrap model with spin proposed earlier by Chiu and Heinman, Dr. Chiu further investigated this model and clarified the assumptions required to give rise to shrinking peripheral peaks.

Another problem investigated in this year is in collaboration with Prof. E. C. G. Sudarshan and Dr. B. Misra. It is on the time development of an unstable quantum system based on the Lee model. In this work, a characteristic time scale prior to the onset of the exponential decay was discovered, which provides an important clue toward the clarification on the long standing 
Zeno's paradox for unstable particle decay.

During 1976-1977, Dr. Chiu's research was in three areas: the strong interaction physics, space-time transformation and special relativity, and formal quantum theory. The bulk of his research is in the first area. Dr. Chiu together with Dr. D. Tow and Mr. M. Hossain continued his work in the dual bootstrap program. They investigated the momentum transfer dependence of Regge trajectories obtained from bootstrap model and further investigated the phenomenological ramifications of their solutions. Dr. Chiu together with Dr. S. Matsuda found an interesting way to correlate multiparticle productions to the intermediate states of the Pomeron diagram in the dual resonance model. Dr. Chiu, together with Professor G. Bialkowski and Dr. D. Tow, proposed a new space-time description for hadron-nucleus collisions. In this new picture, particles at the instance of creation are immature and their maturity rate is enhanced in the presence of other hadronic matter, such as within a nucleus. The model has been very successful. With its only parameter -- the mean maturity time, the model accounts for varieties of data in hadron-nucleus collisions. Dr. Chiu, together with Professor T. E. Kalogeropoulos and Professor E. C. G. Sudarshan showed that existing data confirms our theoretical expectation that the intrinsic parity of the antiproton is negative. Dr. Chiu also furthered his investigation in hydrodynamical model. Using the technique of the information theory, he with Professor C. S. Lam and Dr. K. H. Wang derived equations of motion of hydrodynamical model from a bag-like Lagrangian.

In the second area, Dr. Chiu, together with Dr. J. P. Hsu and Dr. T. Sherry, has studied problems related to space-time transformation. Within the framework of the special theory of relativity the speed of light signals measured by two stable clocks properly arranged is predicted to vary due to the motion of the earth. They further investigated a formalism previously pioneered by Dr. Hsu -- a new space-time transformation based on the notion of the universal time. In the third area, as a continuation of his interest in unstable quantum system, he, together with Professor E. C. G. Sudarshan and Dr. V. Gorini, developed a generalized quantum theory in which one identifies decaying states as complex energy eigenvectors. They also found interesting correlations between the small time behavior of the unstable system with the high energy behavior of the spectrum of the system. 
During 1978-1979, Dr. Chiu continues his research in dual unitarization bootstrap program. Together with Dr. D. Tow and Dr. M. Hossain, he found a simple solution to the bootstrap equation which satisfies the famous Lee-Veneziano relation. Also, together with $\mathrm{Dr}$. D. Tow, he has investigated the compatibility of the bootstrap solution with the Real/Im data which together with other factors has triggered some later interest of including $B \bar{B}$ intermediate states in the bootstrap program.

Dr. Chiu also continued his interest in interactions involving nuclear targets. He, together with Professor E. C. G. Sudarshan and Mr. Prashant Valanju demonstrated how the notion of quantum zeno paradox may be applied to hadron-nucleus collisions. In their work they have also considered finite size effect in hadron-nucleus collisions. The latter consideration has now provided a possible link between their model and the parton model. Together with Professor G. Zinovjev and his collaborators in Kiev, Dr. Chiu has" investigated a fireball model for baryonic inclusive spectra in hadron-nuclei and nuclei-nuclei collisions at high energies. The model gives reasonable description to the available data.

During this year, his new interest in research has been in the direction of QCD, parton model and QCD jets. On the theoretical side, he, Dr. R. Kaul and Dr. E. Takasugi have studied the solution for meron configurations which has some bearing to the understanding of confinement related problem. In the direction of parton model; with Dr. E. Takasugi and a student, $x$. Tata, he studied the problem of multiquark structure function. Within the model, conservation of momentum is strictly imposed. This enables them to investigate the constraint of conservation of momentum in the parton model.

Since August 1978, Dr. Chiu spent his one year leave at Max Planck Institute in Munich. During his stay at MPI, he has been very interested in the nonperturbative QCD problem and small $\mathrm{p}_{\mathrm{T}}$-component of jets. He and Professor Nussinov have investigated the problem of $q \bar{q}$ pair production based onfeynman diagram approach. This work enabled him later on to extract further phenomenological implication of the flux tube model and to demonstrate the agreement of the flux tube model prediction and the $e^{+} e^{-}$data.

Dr. Chiu and Dr. J. Szwed observed that if one assumes that the confinement mechanism sets in after some finite color separation, one should 
expect a two component picture for the quantum-chromodynamic jets: the angular component and the fixed transverse momentum component. The present data is consistent with such an interpretation.

While at the Max Planck Institute, intrigued by the exciting research in the area of cold neutron physics, Dr. Chiu had also the opportunity to collaborate with Professor Leo Stodolsky in the investigation of the feasibility of building a gigantic neutron interferometer using cold neutrons. In the course of this investigation, they discovered a theorem, which among other thịngs, neatly demonstrates the theoretical reason behind those cancellations hitherto noticed in different matter wave interferometry.

Since his return from Germany in Nugust 1979, Dr. Chiu and Dr. Don Tow have been collaborating with physicists in Peking, China, Professor He Zouzlu and his colleague lina wei-qin on a simple space-time description of hadron-nucleus collisions at high energies. The model is based on the twosheet description of soft multiparticle production in hadron-hadron collisions, and can be formulated in a parton framework. This zero parameter model agrees well with the general features of hadron-nucleus data.

In the process of generalizing their model to hadron-nucleus collisions with arbtrary projectiles, Chiu and Tow recognized that their previous model is a special case of a more general model hased on the ideas of: thc Dual Topological Unitarization Theory (DTU) and the confinement string together with estimates on the rate of the breakup of the strings based on for instance the Schwinger formula. This subsequently prompted Chiu and Tow to discover an improved formulation of the DTU-parton model for hadron-nucleus collision. This model leads to the prediction of an approximate $\bar{v}$ universality.

During 1980-1981, Chiu's research was mainty in three areas. The first area is in hadron-nucleus collisions. Chiu further clarified the relationship between their hadron-nucleus collision model, Dual-Topological-Unitarization scheme and OCD confinement. This was presented at the XXth Interrationa) Conference on High Energy Physics at Madison and at the Conference on partons in soft-hadronic processes, Erice.

The second area is in collaboration with Professor Sudarshan, Dr. Chiang and his student $X$. Tata. They investigated the alleged equivalence between Yukawa-type theories and four-fermion interaction theories. Based on this equivalence premise, some authors have constructed the Yang-Mills Theory, the 
Weinberg-Salam Theory and. the $\mathrm{SU}_{5}$ grand unified theory from four-fermion interactions. Chiu and collaborators investigated the corresponding situation within solvable models: the Lee model versus the separable potential model. They found that in a certain strong coupling limit, at finite energies the two models are equivalent; however, their spectra differ at infinity. Thus in the strong coupling limit, by ignoring the spectrum at infinity, Yukawa theory is transmutated into the separable potential theory. Their investigation was carried out in the $N \theta$ and also the $N \theta \theta$ sectors of the models with $N$ and $\theta$ being both bosons. They have also extended their conclusion to the case where both $\mathrm{N}$ and are fermions. Subsequently for the boson case they have further shown that their conclusion is valid in arbitrary sectors. In a separate investigation, they found that the compositeness conditions required for an approximate equivalence between $Q C D$ and the corresponding four-fermion theory may be demonstrated for a large class of gauges.

The third area is in collaboration with Professor Dai of the Chinese Academy of Science, Beijing. They investigated composite models of quarks" and leptons in which the constituents of leptons participate in QCD inter-. action. The constraints from flavor singlet axial vector neutral current, : from cosmology and also ramifications of leptonic gluon emissions were studied. The ultimate detection of color leptons are conjectured.

During 1981-1982, in collaboration with Professor K. C. Chou of Beijing, China and Dr. S. P. Chia of Malaysia, he found that the recently proposed no-go theorem of Weinberg-Witten does not necessarily rule out the possibility of gauge bosons being composite objects. They were able to construct a dynamical model for the composite SU(3) gauge bosons.

Collaborating with Professor. Sudarshan and Dr. Misra, he further investigated the problem of the time evolution of unstable quantum systems. They observed that while the Zeno phenomena is quite relevant in some condensematter systems such ás in nuclear media, its effect on proton decay is negligible. This is despite the fact that the life time of proton is $\gtrsim 10^{31}$ years.

In the same period Dr. Chiu together with Professor Q. B. Xie of Shandong University, Shangdong, China demonstrated that multiparticle pro- 
duction processes in hadron-hadron collisions can be understood semiquantitatively by separating the final-state hadrons into two parts: the two leading particles and the remaining system. The small momentum-transfer hadronization process of the remaining system is assumed to be similar to that of the corresponding processes in $\mathrm{e}^{+} \mathrm{e}^{-}$collisions.

During 1982-1983, Dr. Chiu was interested in problems related to lattice gauge theory and strong interaction dynamics. In collaboration with Helen Quinn and Pisin Chen at SLAC, he studied the vacuum structure of a nonabelian gauge theory with scalars and quarks being the fundamental fields on lattice. Within the mean field approximation, they found that in the strong coupling domain, the chiral symmetry is spontaneously broken. This leads to an extension to an earlier senario. This extension says that composite models of subquarks and leptons based on fundamental fermions and scalars should not be gauge theories of the usual-nonabelian-type.

Dr. Chiu and his student Steve Eubank have been investigating the Migdal recursion relation in the lattice gauge theory. They showed that the string tension on the lattice system can be extracted via Migdal recursions. This work further demonstrates that this recursion relation may serve as a complementary approach to the elaborate and costly Monte Carlo methods.

In multiparticle production dynamics, Dr. Chiu reassessed the role of triple Reggeon interactions at high energies. He observed that there are compelling experimental evidence for triple Regge interactions. He then pointed out that the region with Feynman. variable $x \quad 0.8$ is a unsafe region for the models, such as the recombination model and the counting-rule model. During this period Dr. Chiu and his student Sanford Wilson have also explored the phenomenological and theoretical ramifications of Reggeon fleld theory. 
Or. Gleeson's research interests have been in the general area of elementary particle formalism and phenomenology. Although many of the investigations, such as, studies of kinematic effects and different direct phenomenological data fittings are worth while in their own right, this review will treat only the most significant research accomplishments.

His thesis (University of Pennsylvania 1965) was a detailed calculation of the process of single pion production processes using the analytic S-matrix method. It was and is the most complete ab intio calculation of this process undertaken. It is the only treatment of this problem in which the threshold cuts which arise from unitarity constraints are handled appropriately.

From 1965 to $1969 \mathrm{Dr}$. Gleeson was on the staff of the Physics Department at Syracuse University. While there as both a postdoctoral research associate and as an assistant professor, he developed several new research. topics. In a paper titled "Weak Axial-Vector Currents and the Baryon Field" he was the first to propose and test the chiral algebraic structure of the baryon fields. This paper is based on an extension of the methods developed for the Algebra of Currents by Adler and Weisberger. This analysis is a first step in the identification of the chiral structure of the fermion systems and preceeded by a few years the subsequently developed general chiral symmetric methods for the analysis of strong interactions.

In "Non-invariance Group by Bootstrap and Regge Behavior", Dr. Gleessn! with R. Musto developed and applied a method, later to be called finite energy sum rules, for the investigation of the algebraic structure of meson baryon coupling strengths. This paper is important as regards both its method and intent. The method of finite energy sum rules was later applied by others to solve the problem of balancing the resonance pole versus Regge pole saturation of the intermediate energy cross sections and led to the concept of duality in the strong interactions. The intent was the first use of high energy or Regge behavior to constrain the low energy coupling strengths or more generally to extend the algebraic system in which to express the strong interaction coupling strengths. 
As mentioned above the application of finite energy sum rules to phenomenology of intermediate energy amplitudes lead to the hypothesis of duality. Am amplitude which exactly satisfies the constraints of duality was independently proposed by Gleeson and Meggs in "Infinite Sequence of Resonance Poles and Regge Asymptotic Behavior" and by Veneziano. This amplitude walled the Veneziano representation has served as the classic example for the analysis of an exact dual model and provided the basic framework for the development of dual models of the strong interactions.

In a series of papers, Gleeson, Meggs and Parkinson prôposed tests of several alternative possibilities to the complete duality that was the current style. The present theoretical difficulties in formulating a realistic dual theory have subsequently made most of the discussion of these questions moot, al though they were important at the time.

With Professor Sudarshan, Dr. Gleeson worked on the formulation of a reasonable complete physical interpretation of complex energy negativemetric theories. This work dovetailed nicely with a new interest in this topic brough about by a new model by T. D. Lee and G. C. Wick which used these methods to regulate relativistic field theories. Dr. Gleeson and Professor Sudarshan with collaborators showed in "Analyticity Covariance, and Unitarity in Indefinite Metric Quantum Field Theories" that the LeeWick program was untenable.

In the past few years Dr. Gleeson has initiated with R. Bowers a program for the development of a realistic model of superdense matter. This model when applied to cold systems such as neutron stars, is the only model containing the full complement of baryon constituents which produces star models sufficiently rigid and yet causal which satisfies the present astronomically obtained bounds for mass and.moment of inertia for these objects. These models of superdense matter have also been applied to relativistic heavy ion collision. A model based on an expanding evaporating ball of hot relativistic nuclear-matter has been used to analyze the currently available data on high energy heavy ion collisions. 
RESEARCH HISTORY

Duane A. Dicus - Associate Professor of Physics

While a graduate student at U.C.L.A., Dr. Dicus collaborated with Dr. Ernest Abers and Dr. Richard Norton in calculating the electromagnetic corrections to the weak vector coupling constant of hadrons. This problem had intrigued particle physicists for ten years because of its bearing on the question of the universality of the muon and the hadron weak decay constants. These authors showed the radiative correction to hadron decay was independent of the strong interactions of the hadrons and thus could be calculated by known methods. In a second part of his thesis work, Dr. Dicus discussed the universality of nuclear weak vector coupling constants among themselves. An improved calculation of one of the Coulomb corrections was instrumental in bringing the various coupling constants closer together and resolving the so-called "Al ${ }^{26}$ anomaly".

From 1969 to 1971 Dr. Dicus was a research associate at M.I.T. While there, he, Professor-Roman Jackiw, and Professor Vigdor Teplitz showed that light cone commutators were the natural commutators to use to derive fixed mass sum rules on the structure functions of deep inelastic scattering. This method of deriving sum rules removed ambiguities that existed when equal time commutators were used and some of the old sum rules were corrected. Extending this method in a series of papers over the next several years Dr. Dicus, sometimes with Dr. Teplitz or Dr. David Palmer, derived new sum rules and relations among the structure functions.

Also while at M.I.T. Dr. Dicus, together with Dr. Teplitz and Dr. Daniel Freedman, wrote two papers on the Reggeization of field theories. This work has recently been useful to other investigators in their attempts to Reggeize unified gauge theories.

From 1971 to 1973 Dr. Dicus was a Research Associate and part-time Assistant Professor at the University of Rochester. Other than the light cone work mentinned above, he spent a large share of that time trying to find tests of unified gauge theories. This led him to calculate stellar energy loss rates, various lepton processes, and the radiative corrections to the process $\mathrm{e}^{+} \mathrm{e}^{-} \rightarrow \mu^{+} \mu^{-}$. 
In $1973 \mathrm{Dr}$. Dicus anticipated that the spectrum of hadrons should be SU(4) rather than SU(3) and he and Vishnu Mathur initiated a program of studying the symmetry group chiral SU(4) $\otimes S U(4)$ and how it is broken. Later in 1973 Dr. Dicus joined the University of Texas as an Assistant Professor and he and Dr. N. G. Deshpande continued the work on SU(4) SU(4). This work preceded by more than a year the interest in SU(4) precipitated by the discovery of the $\psi(J)$ particles.

Attempts to find tests of weak interaction theories has been extended to models which are not gauge theories. Dr. Dicus, Dr. Teplitz of Virginia Polytechnic Institute, and Dr. Gino Segre of the University of Pennsylvania have discussed in detail tests of a model based on the exchange of heavy scalar bosons and have made interesting predictions for the processes $\nu_{\mu}+e \rightarrow \nu_{\mu}+e$ and $e^{+} e^{-} \rightarrow \mu^{+} \mu^{-}$. Also, Dr. Dicus has discussed again the use of stellar energy loss rates to test different theories, a method he first used In 1972 preceeding by three years the current interest in such tests.

In addition to his work on finding tests of weak interaction models Dr. Dicus has also been active in constructing improved versions of gauge theories. In particular, Dr. Dicus, Dr. Teplitz, and Dr. J. E. Young of M.I.T. have their own unified gauge theory which uses the strong interaction technique of duality to incorporate the electron-muon universality in a natural way. This model also gives interesting predictions for $\nu_{\mu}+e \rightarrow \nu_{11}+e$ and $e^{+} e^{-} \rightarrow \mu^{+} \mu^{-}$.

The discovery of charmed mesons $\left(D^{0}, D^{+}, D^{*} 0, D^{*+}\right)$ and the realization that their branching ratios depend sensitively on the precise $D^{+}-D^{0}$ and $D^{{ }^{*}}-D^{*_{0}}$ mass differences led to many attempts to calculate these mass differences. Dr. Dicus, together with Dr. Desphande, Dr. Teplitz, and Dr. Ken Johnson of M.I.T., used the M.I.T. bag model to do the first fully dynamical and fully relativistic calculation of these mass differences as well as all other mass differences between particles with the same isospin in the lowest $\mathrm{SU}_{4}$ multiplets of pseudoscalar, vector, spin 1/2 baryons, and spin $3 / 2$ baryons. Later the same group used the bag model to calculate all the Ml radiative transitions between lowest $\mathrm{SU}_{4}$ multiplets. 
In 1973 Dr. Dicus and Dr. Mathur established upper bounds on the masses of particles that occur in unified gauge theories (such as the Higgs mesons). This work was four years ahead of its time; in 1978 Ben Lee and collaborators repeated it and the importance of such bounds were realized.

Currently there is a lot of interest in the overlap between particle physics and astrophysics. Although.Dr. Dicus has been doing such work since 1972 the current interest is at least partially due to his work in 1977 with Dr. Kolb and Dr. Teplitz on the cosmological implications of heavy leptons. They were able to limit the density, masses, and lifetimes of such particles and also the masses of axions and Higgs particles.

Dr. Dicus is part of a group that has analyzed low energy $\pi \mathrm{N} \rightarrow \pi \pi \mathrm{N}$ data. In particular they have compared the older bubble chamber data and the newer results from the single arm spectrometer experiment with current algebra and partial wave isobar model predictions. The most important of the many conclusions is a value for the symnetry breaking parameter of $-0.2 \pm 0.3$. 


\section{S. Nandi - Research Scientist Associate II}

S. Nandi started his research career in 1972 as a graduate student at the University of Chicago, and also as a research assistant in the Enrico Fermi Institute. In 1973, together with Professor Peter G. 0. Freund, he worked on the classic problem of the decay of neutral pion into two photons. The correct decay rate for $\pi^{0} \rightarrow \gamma \gamma$ can be obtained by using Partially Conserved Axial Vector Current (PCAC) or by using Vector Meson Dominance (VMD). Freund and Nandi dryued, by using duality that the two apparantly unrelated pictures for the decay process are equivalent; and explored the consequences by equating the amplitudes obtained via PCAC and VMD. It was found that the strong coupling constant square, $9 \frac{2}{q q \pi}$ comes out to be inversely proportional to the number of color of the quarks. (The assumption $g_{\text {strong }}^{2} N=$ constant, has been the starting point in analyzing the large $\mathrm{N}$ behavior of $\mathrm{SU}(\mathrm{N})$ gauge theory, in perturbation theory, by ' $t$ Hooft et al). Then they argued that the strong interaction is strong because the quarks appear only in three colors. Using SU(6) ${ }_{\mathrm{W}}$ and also KSFR relation, it was further found that $g_{\rho \pi \pi / 4 \pi}^{2} \sim 2.8$, in excellent agreement with experiment.

In 1973-74, S. Nandi, together with Professor Peter G. O. Freund worked on the phenomenology of large transverse momentum exclusive processes. Following the scaling laws proposed by Brodsky and Farrar, and using the compatibility between the scaling and Regge description in the Kinematic region where both applied, they were able to determine the angular distribution of the exclusive hadronic processes. The calculated angular distributions are in good agreement with the available PP, $\overline{P P}, \pi P$ and KP data.

S. Nandi obtained his Ph.D. degree in 1975 from the University of Chicagu. His lhes is work involved the study of supersymmetric quantum field theories. He made an effort to understand the supersymmetry, introduced by Wess and Zumino, dynamically using the requirement of renormalization rnmpatibility. The Wess-Zumino Lagrangian for the fundamental supermultiplet containing a Majorana spinor field $\psi$, a scalar field $\phi$ and a pseudoscalar field, $\phi^{\prime}$ was derived dynamically, starting with the most general renormalizable Lagrangian for these fields, satisfying parity conservation. 
The assumptions underlying the proof are: (i) the bare masses of the fields are equal, and remain so after renormalization; (ii) there exist relations among the bare coupling constants and these relations remain preserved after renormalization. This idea was applied by Drs. D. Wallace and R.K.P. Zia to other renormalizable field theories.

In 1975-76, Dr. Nandi worked at the Enroci Fermi Institute as a research. associate, under the general guidance of Professor $Y$. Nambu. At this time, he got interested in the phenomenology of deep inelastic leptonic processes. Neutrino, electron and muon inclusive data were used by him to understand the structure of the weak currents and also the number of quark flavors.

In 1976, Dr. Nandi joined the Department of Physics, the University of Bonn, West Germany as a visiting scientist. He continued his work on the phenomenology of deep inelastic lepton scattering. With Mr. H. R. Schneider, he estimated the multimuon production in deep-inelastic muon-nucleous scattering. With Dr. G. C. Branco, he analyzed the available neutrino data to determine the structure of the weak neutral currents within the framework of SU(2) $X U(1)$ gauge theory. $V-A$ structure of the weak leptonic current was also analyzed in the light of then available multimuon data in deep inelastic neutrino scattering (in collaboration with Dr. G. C. Branco, Mr. H.P. Nilles and Dr. V. Rittenberg). In Bonn (1976-78), Dr. Nandi also worked on the phenomenology of strong interaction. He (in collaboration with $\mathrm{Mr} . \mathrm{H} . \mathrm{R}$. Schneider and Dr. V. Rittenberg) applied the quark-parton model to understand the general features of the inclusive low $\mathrm{p}_{\mathrm{T}}$ hadronic processes. Quark and gluon fusion model was shown to give good fit to all the inclusive hadronic data. The gluon fusion mechanism was predicted to be the dominant one for $\psi$-production at Fermilab and ISR energies, and the ratio of the yield of $\psi$ in $P-P$ and $\bar{P}-P$ scattering was predicted as a function of energy. This was later confirmed by the Chicago-Princeton group at Fermilab. Also, the available data on $\psi$-production were used by Nandi and Schneider to extract the gluon distribution inside the proton. The gluon distribution was found to be, $G(x) \sim \frac{l}{(n+7)}\left(\frac{1-x}{x}\right)^{n}$ with $n=5 \pm 1$; in good agreement with the data at this time.

While in Bonn, Dr. Nandi (in collaboration with Dr. W. C. Ng) worked the general problem of coupling constant relations in renormalizable field theories. Using renormalization group approach, they analyzed the con- 
ditions in which several coupling constants, in a field theory, can be related to each other; the relations being independent of the choice of the renormalization point. In particular, they investigated the criteria for the feasibility of a power series relations among the various coupling constants, in various theories. It was shown that such a power series relation exists, order by order in perturbation theory, in spontaneously broken gauge theories (with Higgs scalars) when the gauge group is a simple one. Application to the Weinberg-Salam model (including Higgs scalars), gave, in lowest order, the Higgs mass to be $\sim 240 \mathrm{GeV}$.

From 1978-80, Dr. Nandi was at the Ohio State University as a research associate. He worked in the area of $S U(5)$ and $S O(1 \cap)$ Grand Innified Theories, fermion masses and mixing angles in gauge theories, and also in the application of the QCD perturbation theories to the jet. strurcture in $\mathrm{e}^{+}-\mathrm{e}^{-}$ annihilation, and in hadronic p-p scattering.

In their work in OCD, Dr. Nandi and collaborators calculated the quantum-chromodynamic correction to jet (defined in the manner of Sterman and Weinberg) angular distribution in energetic $\mathrm{e}^{+} \mathrm{e}^{-}$annihilation to order $\alpha_{s}$. Keepint exact dependence on $\varepsilon$ and $\delta$, they found deviations from the zeroth-order distribution, $1+\cos ^{2} \theta$ for relatively large values of $\varepsilon$ and $\delta$ (See S. Nandi and W. W. Wada, Phys. Rev. D21, 76 (1980)). This effect could be tested in the existing $e^{*} e^{*}$ colliding beam facilities. They also studied the gluon jets in proton-proton collisions, using a charmonium modei (See G.D. Arbrecht, II, S. Nandi and W.W. Wada, Phys. Lett. 86B, (1979) 79).

During his stay at Ohio State University, Dr. Nandi also worked on Grand Unified Theories (GUT). His work involved: the understanding of the observed relations among the masses of quarks and leptons using SU(5) and SO(10) GUT and calculating the mixing angles among the fermions which govern their productions and decays in weak processes; calculating neutrino masses and mixings in SO(10) relevant for neutrino oscillations experiments; and attempting to understand the observed replication of fundamental fermions in the form of families. Presently he continues his research in these directions.

Quark-lepton mass relations and mixing angles were studied using SU(5) and SO(10) GUT together with discrete symmetries. The Georgi-Jarlskog mass relations $m_{\mu} / m_{e}=9 m_{s} / m_{d}, m_{\tau}=m_{b}$ were obtained using two $\underset{\sim}{5}$ and one $\underset{\sim \sim}{45}$ 
Higgs representations and a discrete symmetry. The Kobayashi-Maskawa mixing angles were found to be $s_{2}=\left(m_{c} / m_{t}\right)^{\frac{1}{2}}$ and $s_{3}=\left(m_{u} / m_{t}\right)^{\frac{1}{2}} / s_{1}$ where $s_{1}=$ sine of the Cabibbo angle. The b-quarks were predicted to decay predominantly into $c$-quarks with lifetime of $\tau_{b} \cong 10^{-13} \mathrm{sec}$ (see $\mathrm{S}$. Nandi and $\mathrm{K}$. Tanaka, Phys. Lett. 92B, 107 (1980)). Mixing angles and CP-violation was also studied in $S U(2)_{L} \times U(1)$ gauge model supplemented by permutation symmetry $\mathrm{S}_{3}$ for six quarks (see $\mathrm{S}$. Nandi and K. Tanaka, Phys. Rev. D21, $2684(1980))$.

Recent experiments indicate that neutrinos may have masses of the order of ev, with large mixing among themselves. In SI(10) GUT, since there is an extra neutral lepton, neutrino mass arises naturally. Nandi and collaborators assumed that the left-handed neutrino acquires a small mass via the Gell-Mann-Ramond-Slansky mechanism. They then computed the neutrino mixing angles in terms of the mass ratios of up-quark families the SO(10) gauge model supplemented by a discrete symmetry. They found large $\nu_{\mu}-\nu_{. \tau}$ mixing (see S. Hanna, K. Milton, S. Nandi and K. Tanaka, DOE-ER-1545-282; Phys. Lett. $B$, in press)).

One outstanding problem in particle physics is the replication of fundamental fermions in the form of the $\left(5^{*}+10\right)$-plet of SU(5) families. In their study of fermion mass matrix using 45 representation of Higgs, Nandi and collaborators found that the number of such families has to be six in order to obtain acceptable quark-lepton mass ratios (see P.H. Frampton, S. Nandi and J.J.G. Scanio, Phys. Lett. 85B, 225 (1979)). Also, following the guidelines suggested by $H$. Georgi, they. proposed a SU(9) superunified model which contained three to six SU(5) families naturally, and the mass relations among the fermions of different families were obtained. The guiding principle was to minimize the number of Higgs scalars necessary to obtain mass relations (see P.H. Frampton and S. Nandi, Phys. Rev. Lett. 43, 1460 (1980)).

Starting September 1, 1980, Dr. Nandi joined the Center for Particle Theory. He continued his work on Grand Unified Theories, especially on predictions of such theories at low energy, such as proton decay, family unification, neutrino oscillations and the presence of a small admixture of $V+A$ charged weak current along with the dominant $V-A$. 
In $S 0(n)$ family unification schemes, it is well known that for every family contained in the $S O(n)$ spinor representation, there exists a corresponding conjugate family. The particles belonging to the conjugate family have $V+A$ weak interactions. Dr. Nandi, Dr. Stern and Prof. Sudarshan (Phys. Rev. D26, 2522 (1982)) pointed out that the mixing between the particles in a usual family with those of a conjugate family induces a $V+A$ admixture into the. usual low energy weak interaction. In applying this to pion and muon decays, they found that although the data is in very good agreement with $\mathrm{V}-\mathrm{A}$ theory, a certain amount $(\sim 10 \%)$ of $\mathrm{V}+\mathrm{A}$ admixture can be tolerated. In fact, some of the mixing angles may be arbitrarily large. They considered both the cases where conjugate neutrinos are either light or heavy.

Dr. Nandi continued his work on neutrino mixing. In an earlier work, he and his collaborators pointed out that in SO(10) grand unified theory, the mixing between $v_{e}$ and $\nu_{\mu}$ is expected to be very smali $\left(\sim \theta_{c}\right)$, whereas that between $\nu_{\mu}$ and $\nu_{\tau}$ can be large. This is essentially due to the hierarch of the quark masses. In a further work, Dr. Nandi (with K. Milton and K. Tanaka, Phys. Rev. D25, 800 (1982) illustrated quantitatively how the. parameter in the Majorana sector must be tuned in order to achieve a large $v_{e}-v_{\mu}$ mixing in a $S 0(10)$ theory.

The most important prediction of grand unified theories is the decay of proton and bound neutron with a possible observable rate. As a continuation of their work on proton decay, Dr. Nandi, Dr. Stern and Prof. Sudarshan (Phys. Lett. 113B, 165 (1982)) examned the question of whether the yauge interactions leading to proton decay can be rotated away from the grand unified models via a suitable choice of the flavor mixing alngles. Grand unified models with an arbitrary number of families and an arbitrary gauge group was considered. In the case of the SU(5) model with three generations, it was shown that the proton (and bound neutron) decay could be rotated away only if $S_{3}=0$ where $S_{3}$ is the sime of the third Kobayashi-Maskava mixing angle. This has very interesting implications in bottom quark decays and also in CPviolation.

During the last year, Dr. Nandi worked in both globally and lucally supersymmetric unified theories. 'These works' are reported in lie accompanying progress report. 


\section{RESEARCH HISTORY}

Xerxes R. Tata - Research Associate

Dr. Tata joined the Center for Particle Theory, Physics Department, University of Texas at Austin in midsummer 1978 as a student working under the supervision of Dr. Charles Chiu. He graduated in Summer, 1987. He now holds a joint appointment as a Research Associate in the Center for Particle Theory and The Theory Group, University of Texas at Austin.

Drs. C. B. Chiu, E. Takasugi, R. Kaul and X. Tata have developed a model, based on the recombination mechanism suggested by Das and Hwa, to describe small $p_{T}$ particle production in high energy hadron collisions. Dr. Takasugi and Dr. Tata have applied this model to inclusive single meson, single baryon, multimeson and associated meson-baryon production in the fragmentation domains of the proton, the pion and the kaon. Their. parameter free predictions for momentum correlations in inclusive multiparticle reactions are in good agreement with a fair amount of recently obtained correlation data.

Dr. Tata, together with Drs. Chiu, Chi.ang and Sudarshan has also been working on the problem of equivalence of the four-fermion interaction and the corresponding Yukawa type interaction. They have shown, in the. context of the soluble Lee model and the separable potential model, that although it may be possible to satisfy the so-called compositeness conditions, the equivalence of the two theories does not necessarily follow. They have gone on to show that at least in their model study the Yukawa interaction may be transformed into a four-point interaction provided certain spectral contributions that occur in the Yukawa theory are explicitly omitted. Drs. Chiu, Chiang, Sudarshan and Tata have demonstrated that the transmutation of the Yukawa interaction into a four-fermion interaction can be demonstrated in a general fashion using operator methods. In their study of the corresponding situation for fully relativistic field theories, they have shown that for quantum chromodynamics with SU(3) for the colour group, it is possible to satisfy the compositeness conditions if the number of flavours is between 
ten and sixteen. The question of equivalence with the corresponding four fermion interaction is still open.

Dr. Tata, along with Drs. Chiu and Chiang, has also studied the work of Durr and Saller, who, following Heisenberg, have attempted to derive all the physical particles as composites of one single quartically self-coupled spinor field. They conclude that Durr and Saller have not quite demonstrated that it is possible, by dynamical rearrangement, to a.7ter the symmetry properties of a Lagrangian system.

Dr. Tata and Prof. Sudarshan have constructed à mode 1. quantum field theory in three space dimensions for which the complete spectrum of the Hamiltonian could be exactly obtained. Furthermore. all the wave functions, and hence the S-matrix elements can be obtained using a recursive procedure.

Ur. Tata, Prof. Sudarshan and Prof. Joseph Schechter (Syracuse University) have pointed out that a spontaneous breakdown of global supersymmetry is consistent with unbroken Poincare invariance if and only if the SUSY algebra." $=0 "$ is understood in the weaker sense $[, \phi]=0$ for a 11 the dynamical variables $\phi$ rather than as an operator equation. They point out that this does not alter any of the conclusions about supersymmetric quantum field theories that have been obtained using the strong form of the aigebra.

In a separate paper, Prof. Dicus and Dr. Tata have shown that finite temperature effects do not necessarily. break the SUSY Ward-Takahashi identities. They point out, however, that the physical significance of these identities is not clear since they involve a grading $(-1)$ for fermions and +1 for bosons.

In recent months, Dr. Tata has been involved in studying ways to experimentaliy detect supersymmetry. In collaboration with Prof. Dicus and or. Nandi he has studied the decay of the $W$ busun into supersymmetric particles, and further, the subsequent decay of these particles within the framework of a supersymmetric unified theory in which gravity breaks. SU(2) $L U(7)_{Y} \rightarrow U(1)_{E M}$ : They havc pointed out that if the decay of the $W$ boson into a wino and a zino is kinematically allowed, the subsequent decays of the ganginos may provide a definitive signature of SUSY. 
Continuing their search for ways of experimentally detecting SUSY, Dr. Dicus, Dr. Nandi, Dr. Tata in collaboration with Dr. W. W. Repko (of Michigan State University) have studied signatures for supersymmetric decay modes of $W$ that would be expected to be seen at the CERN $\bar{p} p$ collider. They concluded that if a wino with a mass less than $50 \mathrm{GeV}$ exists, it will show up in the CERN data. They note that a dequential heavy lepton of comparable mass would, however, be indistinguishable from the wino.

In a separate paper, Dr. Tata along with Dr. Dicus, Dr. Nandi and Dr. Repko have studied the production of SUSY particles by electro-positron collisions. They have concluded that for a wide range of masses of the wino and supersymmetric scalars, a measurement of the total $\mathrm{e}^{+} \mathrm{e}^{-}$annihilation cross section is sufficient to demonstrate the existence or nonexistence of SUSY. The signatures they discuss are expected to be seen at LEP but if the wino or scalar masses are smal1 enough, these may be seen much earlier at SLC.

Dr. Tata, who will be moving to the University of Oregon in Fa11, 1983 will be continuing his studies of supersymmetric field theories. 


\section{RESEARCH HISTORY}

\section{Allen Stern - Postdoctoral Research Associate}

From 1975 to 1979 A. Stern was a Research Associate at Syracuse University under the guidance of Professor A. P. Balachandran. The topics which he investigated were charge monopole scattering, dynamical systems on group manifolds and dual string models and their interactions.

Regarding the first topic, charge monopole scattering, Stern (with A. P. Balachandran, S. Borchardt, S. S. Chang, R. Cahalan, R. Ramachandran and $H$. Rupertsberger) derived a semiclassical approximation (starting from the Feynman path integral) for the electric charge-maqnetic monopole differential cross section. The problem was non-trivial because of the presence of many classical trajectories at large scattering angles. The analyticity of the exact quantum mechanical scattering amplitude was also investigated.

Concerning the second topic, Stern (with A.P. Balachandran and S. Borchardt) found a Lagrangian description for particles with internal spin in interaction with an arbitrary gauge field. The Lagrangian possessed the unique feature that it was defined on a group manifold. This feature was found to have applications for three other problems. In the first problem, the dynamics of a spinning particle interacting with gravity could be formulated on the Poincare group manifold. Along these lines, Stern in collaboration with A. P. Balachandran, G. Marmo and B. Skagerstam developed an action principle leading to the MathissonPapapetron equations. The second problem was concerned with the dynamics of the charge-monopole system. It was found that this system could be formulated on an SU(2) group manifold. This treatment, unlike previous treatment, had the advantage that the action was globally defined and contained no "Dirac string" singularities. This investigation, along with a supersymmetric extension, was carried out in collaboration with Balachandran, Marmo and Skagerstam. In the third problem, the role of group manifolds in the description of non-linear field theories was shown (with Balachandran and G. Trahern). 
Concerning the third topic, A. Stern (with Balachandran and Skagerstam) generalized the previous work on particle interactions to include dual string interactions. Upon coupling strings to gauge theories, the strings were found to have properties similiar to superconductors. Similiar results were found for the coupling of higher dimensional objects (shells and bags). The dynamical system developed above was employed in studies of the QCD vacuum. In collaboration with Skagerstam, Stern demonstrated that the dynamics of a "hole" in a (dual) superconducting 4-volume was identical to the dynamics of gluons in an MIT bag. They also used the dynamical description of charged strings as a model for a "gluering" or giueba17. In this context they showed that glueball bound states can occur in the (Copenhagen) vacuum, al though they are highly unstable.

From September 1980 to the present time A. Stern was a Postdoctoral Research Associate at the University of Texas at Austin. During this period he continued examining the problem of relativistic point particle dynamics. Along with Skagerstam he found a Lagrangian description for relativistic spinning particles interacting with an electromagnetic field which lead to the Bargmann-Michel-Telegdi equation. The above description was free of the difficulties encountered in previous treatments of the problem. In collaboration with A. P. Balachandran, Stern studied certain 2-dimensional field theories which possessed infinitely many conservation laws. They were able to apply some of the techniques used in these theories to the Nambu string model. As a result finite number of new conservation laws were constructed for the string.

While in Austin, Stern collaborated with. Dr. S. Nandi and Professor E. C. G. Sudarshan on three problems concerned with qrand unified models, In the first, they studied the structure of baryon decay within the context of $S O(n)$ family unification schemes. They showed that in the gauge sector of the theory the structure of baryon decay is essentially unaltered upon expanding an SO(10) single family unification model to an $S O(n), n>10$ multifamily unification model. This was essentially due to the presence of certain "conjugate" families inherently contained in all SO(n) models. The second problem concerned the possibility of mixing occurring between the conjugate families and the usual families of $S O(n)$ theories. Such a mixing 
would induce $V+A$ corrections to the usual theory of low energy weak interactions. It was found that although the data is in very good agreement with V-A theory, a certain amount of $\mathrm{V}+\mathrm{A}$ admixture can be tolerated. The third problem dealt with the question of whether or not the interactions leading to proton decay could be rotated away via a suitable choice of flavor mixing angles. For the specific case of an SU(5) model with three generations (and an arbitrary number of. Higgs multiplets) it was shown that proton decay could be rotated away if the sine of the 3rd Kobayashi-Maskawa angle is zero.

In collaboration with A. P. Balachandran, V. P. Na1r and S. Rajeev, Stern studied the interaction of baryons with topological solitons of the $S U(2) \times S U(2)$ chiral model. It was found that soliton states and solitonbaryon bound states have exotic properties; eg. large baryon number and strangeness, fractional charge and an exotic relation between spin and baryon number. Furthermore, the states were found to be long-lived. It was speculated that they may be related to the recentiy observed anomalous nuclei.

During the academic year 1982-83, Stern was on leave from the University of Texas and was at the Institute of Theoretical Physics in Gothenberg (Sweden), There in collaboration with Balachandran, Marmo and Skagerstam he worked on a review article introducing fibre bundles via examples involving point particle dynamics. The exaniles included maynelic munupoles, spinning particles, particles in a non-abelian gauge field and a KaluzaKlein description of point particles. Additional topics considered were (i) the extension to systems involving fermionic degrees of freedom, ( $i$ ) the quantization of the above examples and ( $i i i)$ the general question of how global Lagrangians can be constructed from systems which admit only local Lagrangians. 


\section{RESEARCH HISTORY}

Patrick J. Moylan - Graduate Student

As a student at the University of Texas at Austin, Patrick Moylan became interested in the Mossbauer effect. In his master's thes is he verified the magnetic moment verses spin formula of the metal ion law of chemical compounds for certain chemical compounds $\left(\mathrm{Fe}(8-\mathrm{QS})_{2} \cdot 1 \frac{1}{2}\right.$ $\mathrm{H}_{2} \mathrm{O}$ where $\mathrm{X}$ is an appropriate halogen-1ike compound--Cl, Br, I NCS) using the spectra obtained from his Mossbauer analysis of the compounds.

Working with $A$ : Bohm and others at Texas, he applied a phenomenological theory; based on $S U(4)$ as a spectrum-generating group for the hadrons, to the calculation of the radiative decays of the vector mesons (1977). Recently this theory has obtained amazing success through the work of J. Werle, A. Bohm, R. B. Teese, A: Garcia and P. Kielanowski.

In 1979 he took up a theoretical problem which he worked on for a year. It was to describe the bound and scattering states of the hydrogen atom by a fiber bundle over the configuration space of the center of mass. It is a model for an elementary system (a hydrogen atom), which avoids the use of constituents. The model leads directly to many sophisticated analytical techniques which can be used to prove such results as the completeness and orthogonality of the spherical harmonics on the thre sphere.

During 1980 he investigated classical radiation reaction and the classical Lorentz-Dirac equations of the electron. Specifically he studied classical and semiclassical radiative shifts predicted by these equations of motion of the electron in quantum mechanics as derived by Moniz and Sharp (see Grotch, Kazes et al. in preprint LA-UR-81-1164).

Mr. Moylan's dissertation dealt with a study of the spin of elementary particles. It is experimentally observed that hadrons have a massspin relationship. In his dissertation he investigated . the consequences of a relativistic wave equation for an elementary particle which gives a relationship between the mass and the spin of the particle. The 
equation is:

$$
\begin{aligned}
Q \psi & =\alpha \psi \quad(\alpha=\text { eigenvalue characterizina the system }) \\
\lambda^{2} Q & =B_{\mu} B^{\mu}-\frac{1}{2} \lambda 2_{L \nu} L^{\mu \nu} \quad\left(B_{\mu}=P_{\mu}+\frac{1}{2} \lambda\left(P^{\rho} P_{\rho}\right)^{-\frac{1}{2}}\left\{P^{\sigma}, L_{\sigma \mu}\right\}\right) .
\end{aligned}
$$

$P_{\mu}$ and $L_{\mu \nu}$ are the generators of the Poincare group and $\lambda$ is a constant which is experimentally determined and found to be on the order of the fundamental length of the strong interactions $--10^{-13} \mathrm{~cm}$. The five princibal sections are:

1. Introduction to the DeSitter Group. This section is devoted to a description of the DoSititer group and DeSitter space using concepts from ilber bindle theory. Aside f'rom any new physical insight, the fiber bundle description provides a clear picture of many concepts used in gauge theories. A type of fiber bundle which I consider is one which satisfies the conditions of soldering. ${ }^{1}$ Soldering transfers the meaning of distance from the Minknwski space-time to the fiber over a point in the base space-to wse the lanplape of fiber bundies. Ti: nlso permits the correlation of. the rotations of the $S O(3,1)$ subgroup of the structural group $S O(4,1)$ of the bundle with the physical rotations of the Lorentz group. I use soldered fiber bundles in a matheratical treatment of the hydrogen atom, based on a method due to Fock.

¿. Unitary representations of $00(4,1)$ on 1rreduciblo representations. of the Poincaré group. ${ }^{3}$ ihis part of my dissertation deals with the properties of certain unitary representations of the $(4+1)$ Desitter group, so(4,1). The group has a realization as a subgroup of the group of confornial iransformations of the mornentum space of Minkowskj. space. As a consequence of this realization I have proven a conjecture of Bohm on the intcgrability of a particular realization of the principle series representations of the Lio algebra of $50(4,1)$. $^{4}$ In proving this result I have obtained a simple derivation of the formula, 'which was first obtained by S. Strom, ${ }^{5}$ for the 
decomposition of a UIR (unitary irreducible representation) into UIR's of its non-compact subgroup, $30(3,1)$. As a by-product of the derivation, I have obtained a very simple method to determine the natrix elements of the generators of $S O(4,1)$ in the $S O(4)$ basis.

3. The Desitter Symmetry of the Dirac Equation. This section is based. on the results of the previous section. Here it is shown that the construction of the IIR's of SO $(4,1)$ given above affords us a previously unknown SO $(4,1)$ symmetry of the Dirac equation. ${ }^{6}$ As is well-known, the Dirac i $\Gamma_{\mu}{ }^{\prime} s\left(\Gamma_{\mu}=\frac{1}{2} Y_{\mu}\right)$ and $s_{i N}$ 's close to form an $s 0(4,1)$, but they do not commute with the Dirac equation. I obtain the 10 generators $\frac{1}{\lambda} B_{\mu}$, $L_{\mu \nu}$ of an $\operatorname{So}\left(1_{4}, 1\right)$ which commute with the nirac equation and so is a symmetry group of the equaticn. The physical meaning of the $I_{\mu \nu}$ 's 1 s clear. The meaning of the $\frac{I_{B}}{m_{i}: i}$ 's may bo that of a center-of-mass velocity of the particle.?

An jntwrestine conciequence of these results is that the UIR of So $(4,1)$ constructed in this, way needs the Hilbert space of two irreduciblo unitary representations of the Poincaré froup of mass $m$ and $\operatorname{spin} s$. This doubling of states, for half-integer spin particles, can be associaterl with the antiparticle. ${ }^{8}$

4. The Relativistic Rotator. In this section I describe a relativistic wave equation for an elementary particle which gives a relationship between the mass and the spin of the particle. The equation is: ${ }^{9}$

$$
\begin{aligned}
& n i=\alpha \psi \quad(\alpha:=\text { eigenvalus characteriaing the system }) \\
& \lambda^{2} Q=B_{\mu l} B^{\lambda}-\frac{1}{2} \lambda^{2} L_{\mu \nu} L^{\mu \nu} \quad\left(B_{\mu}=P_{\mu}+\frac{1}{2} \lambda\left(P^{\rho} P_{\rho}\right)^{-\lambda}\left\{P^{\sigma}, L_{\sigma \mu}\right\}\right) .
\end{aligned}
$$


$P_{\mu}$ and $L_{\mu v}$ are the generators of the Poincare group and $\lambda$ is a constant which is experimentally determined and found to be on the order of the fundamental length of the strong interactions--10-13 $\mathrm{cm} .^{10}$ The operator $Q$ is the Casimir operator for the $\mathrm{so}(4,1)$ representation described in the previous two sections. One reason for calling it the relativistic rotator is because eqn. (1) can be derived from two equal mass particles rotating rigidly about each other. ${ }^{11}$

\section{A comparison of vartous theoretical mass-spin relations with} experiment. "As a part ol my dissertation I discuss the experimental agrecment of various mass-spin relations, ${ }^{12}$ in particular the linear relation $m^{2}=\alpha_{0}+\alpha_{1} s$ of Regge theories ${ }^{12}$ and the one given by equation (1), which is $\mathrm{m}^{2}=\mathrm{m}_{0}^{2}+\lambda^{2} \mathrm{~s}(\mathrm{~s}+1)$. I compare these two formulae to the experimental mass spectra of the mesons. It is found that either formula provides good agreement with a certain subset of the meson data. Better agreement is obtained in the rotator model by introducing a perturbative term to account for the change in radius with increasing spin.

In this perturbative modification of eqn.(1), states are described by certain UIR's of $\mathrm{SO}(3,2)^{9}$ which are generalizations of the UIR's obtained by Dirac and Majorana--see Relativistic Models of Extended Hadrons Obeying a Mass-Spin Trajectory Constraint, by N. Mukunda, H. van Dam and L.C. Biedenharn, Springer (1982). 


\section{RESEARCH HISTORY}

\section{Tom Schimert - Graduate Student}

During the past two years, Tom Schimert has been studying, along with Professor Sudarshan and Dr. Chiang, the Appelquist-Carazzone decoupling theorem (Phys. Rev. D17 (1975) which states that given a quantum field theory with two mass scales of very different magnitudes, at energies well below the heavy-mass threshold the effect of the heavy sector in Feynman diagrams contributing to ligh-particle processes are suppressed by an inverse power of the heavy mass scale.

Although the theorem is intuitively plausible it is by no means obvious. This is because the validity of the theorem depends on ones choice of regu-.. larization procedure for the handling of ultraviolet divergence which arise in perturbative quantum field theory. Two common regularization procedures involve what are known as minimal subtraction regularization and momentum subtraction regularization; respectively. It can be shown by explicit calculation to one-loop order that if minimal subtraction regularization is employed the theorem does not hold.

However, if one uses the momentum subtraction scheme, we have shown that the theorem holds for a wide class of theories. More specifically, we have shown (Univ. of Texas preprint DOE-ER-03992-520) that the heavy-mass behavior of an arbitrary Feynman diagram, arising in the perturbative expansion of a quantum field theory with two very different mass scales, is always suppressed by an inverse power of the heavy-mass scale at energies on the order of the light-mass scale, so long as the momentum subtraction scheme is employed, and that these subtractions are carried out at energies on the order of the light-mass scale.

We would like to emphasize, however, that our proof is only valid for theories which do not contain coupling constants which exhibit unsuppressed heavy-mass dependence. What this means is that our proof does not hold for theories in which the two different mass-scales are generated by spontaneous breakdown of an internal gauge symmetry, i.e., the most physically interesting theories. However, work to extend the theorem to theories of this type are presnetly in progress. 
The second area of research Mr. Schimert has benn pursuing, along with Dr. Tata, is phenomenological supersymmetry. The model they are working with is a supersymmetric version of the SU(2) $\times U(1)$ Weinberg-Salam model. It predicts the existence of the superpartner to the $W$ bosons, the winos with a mass less than the $W$ itself; which means that it it exists it should be seen experimentalily in the near future.

They are presently calculating the cross-section for wino-antiwino pairs in $e^{+} e^{-}$collision. They hope that this process will be distinguishable from heavy lepton-antilepton production and hence if observed experimentally would be a signal that supersymmetry is a viable theory. 